\title{
Análisis de las Funciones de Importación y Exportación de México 1980-2000
}

\author{
Daniel G. Garcés Díaz* \\ dgarces@banxico.org.mx
}

Diciembre de 2002

Documento de Investigación No. 2002-12

Dirección General de Investigación Económica

BANCO DE MÉXICO

\footnotetext{
Agradezco a Adalberto González, Rafael Gómez-Tagle, Sara Castellanos, Alejandro Werner, Alfredo Cuevas y a los participantes en seminarios en el Banco de México y en el Encuentro Europeo de la Sociedad Econométrica en Lausana por sus útiles comentarios y sugerencias. Rocío Elizondo colaboró de modo eficiente en todos los aspectos de este documento. El autor es por supuesto responsable de cualquier error $\mathrm{u}$ omisión. Asimismo, el contenido de este trabajo no refleja necesariamente las opiniones del Banco de México.
} 


\title{
Análisis de las Funciones de Importación y Exportación de México 1980-2000
}

Daniel G. Garcés Díaz

dgarces@banxico.org.mx

Diciembre de 2002

Documento de Investigación No. 2002-12

\begin{abstract}
$\underline{\text { Resumen }}$
En este documento se estudian las funciones de largo plazo y la dinámica del comercio exterior de México con datos agregados y desagregados. Las exportaciones totales dependen del índice de la producción industrial de los Estados Unidos y del tipo de cambio real. El nivel de las importaciones totales es función del índice de producción industrial de México, del nivel de las exportaciones totales y del tipo de cambio real. Un resultado importante es que casi todas las funciones, tanto de largo como de corto plazo, son estables para el período 1990-2000 mientras que ninguna lo es para toda la muestra. Esto sugiere que la apertura comercial de los años ochenta provocó un cambio estructural muy pronunciado en el comercio exterior mexicano y que el TLCAN es la continuación natural de dicho proceso.
\end{abstract}




\section{Introducción}

El objetivo de este trabajo es estudiar la evolución de los flujos de comercio exterior de México de 1980 a 2000, período que incluye importantes cambios institucionales tales como la adhesión de México al GATT (1985) y el inicio del Tratado de Libre Comercio de Norteamérica (TLCAN) en 1994. El notable desempeño del comercio exterior mexicano en los últimos años ${ }^{1}$ ha sido un tópico frecuente tanto en discusiones de política económica como en la prensa. Sin embargo, los estudios rigurosos sobre este tema han sido sorprendentemente escasos y se han concentrado en evaluar únicamente los cambios ocurridos en los años noventa.

Gould (1998) encontró que el comercio de Canadá con los Estados Unidos y México no tuvo efectos perceptibles después del TLCAN pero que el comercio entre México y los Estados Unidos si mostró cambios notables. Este autor basó sus estimados en un modelo de gravedad del comercio bilateral donde el cambio en las barreras tarifarias y no tarifarias es aproximado con una variable dicotómica que resultó significativa para las ecuaciones de comercio entre México y los Estados Unidos.

Por su parte, Krueger (1999) señala que tanto el comercio canadiense como el mexicano se han vuelto más concentrados hacia los Estados Unidos e indaga, también en el contexto de un modelo de gravedad, si dicho fenómeno es el resultado de la creación o de la desviación de comercio. La autora concluye que el TLCAN no ha inducido grandes efectos con respecto a otros procesos ya en marcha, tales como la reducción previa de tarifas y la modificación del régimen del tipo de cambio en México. Gruben (2001) encuentra que el TLCAN ha tenido poca influencia sobre el empleo en las plantas maquiladoras mexicanas.

El interés en el estudio de los efectos del TLCAN sobre el comercio exterior mexicano es comprensible por ser este acuerdo uno de los eventos económicos e institucionales más importantes de la última década. Un factor adicional que quizás explique el énfasis en la experiencia reciente es la dificultad de obtener modelos econométricos que cubran períodos largos, debido a posibles cambios estructurales en las relaciones económicas o modificaciones metodológicas en la elaboración de las estadísticas. Por ejemplo, en un sofisticado estudio para muchos países con datos

\footnotetext{
${ }^{1}$ De ese año hasta 2000 las exportaciones crecieron, en promedio, casi tres veces más rápido que durante los seis años previos: $15.5 \%$ contra $5.5 \%$ anualmente. Para el mismo período, las importaciones aumentaron $10.6 \%$ y $18 \%$ en promedio anual, respectivamente. El grado de apertura de la economía, definido como la suma de las exportaciones e importaciones sobre el PIB, para el año 2000 (0.77) es más de dos veces mayor que el de 1993 (0.36).
} 
anuales de 1960 a 1993, Senhadji y Montenegro (1998) no lograron obtener estimados con el signo correcto para la función de exportaciones de México. Esto probablemente obedeció a alguna de las causas mencionadas, ya que su metodología parece apropiada y fue exitosa en muchos otros casos. ${ }^{2}$

En este trabajo se examina más detalladamente la evolución de los flujos comerciales de México suponiendo que éstos están enteramente determinados por el lado de la demanda. Es decir, las funciones de oferta de importaciones y exportaciones son perfectamente elásticas dentro del intervalo observable o, equivalentemente, que el país es un tomador de precios en los mercados mundiales. Este es un supuesto razonable para México.

Debido a la mencionada dificultad de obtener modelos para todo el período 1980-2000, éste se divide en dos submuestras, 1980-1989 y 1990-2000, enfatizando la incorporación de México al GATT y al TLCAN, respectivamente. Aunque se da una relevancia mayor al estudio de la segunda submuestra, también se obtienen modelos de largo plazo para la primera. El análisis se realiza con el uso simultáneo de varios métodos de estimación de relaciones de largo plazo.

Los resultados principales se señalan a continuación. Se obtienen coeficientes con el signo correcto y magnitud razonable en la estimación de funciones de demanda de largo plazo para las exportaciones e importaciones en el período 1990-2000. La ecuación de exportaciones es función del índice de la producción de los Estados Unidos y del tipo de cambio real y resulta muy estable para dicho período. La función de importaciones, que depende del índice de la producción industrial de México, del nivel de las exportaciones y del tipo de cambio real, muestra inestabilidad, aunque la ecuación ajusta los datos muy bien aun sin considerar la estacionalidad o la dinámica de corto plazo.

El análisis se extiende para datos desagregados y para el período 1980-2000 y se obtiene que solamente en unos pocos casos se puede encontrar cointegración para toda la muestra (19802000) y en ningún caso el vector correspondiente es estable. Para el período 1990-2000 se obtuvo un vector de cointegración estable en todos los casos. La inestabilidad de la ecuación de las importaciones totales para la submuestra 1990-2000 no se transfiere a los datos desagregados. También se obtienen modelos de corrección de error estables y constantes para igual período.

En la mayoría de los casos, pudieron obtenerse vectores de cointegración para la submuestra 1980-1989, aunque éstos no pasan las pruebas de estabilidad. Los resultados anteriores indican que si bien un cambio estructural en el comercio exterior de México durante los noventa no

\footnotetext{
${ }^{2}$ Estos autores obtienen buenos estimados en 53 de 70 casos analizados y demuestran que las demandas de importaciones y exportaciones tradicionales se pueden derivar de un marco de equilibrio general.
} 
parecer haber tenido lugar, uno muy importante fue inducido por la apertura de la economía y la incorporación de México al GATT en los ochenta Por esa razón, no es posible, en general, encontrar relaciones estables comenzando desde 1980. La estabilidad de las funciones de comercio exterior obtenidas para los noventa probablemente obedece a que el TLCAN fue el mecanismo necesario para que los beneficios de la apertura comercial se continuaran dando.

El resto del documento está organizado como sigue: en la sección I, se describen los datos y se explora la presencia de raíces unitarias en ellos. En la sección II, se analiza el comportamiento de las exportaciones e importaciones a nivel más agregado. En la sección III, se lleva a cabo el estudio para diferentes tipos de exportaciones e importaciones. En la sección IV, se analizan los modelos de corrección de error. En la sección V, se proporcionan las conclusiones.

\section{Descripción y Propiedades Estadísticas de los Datos}

Los datos de exportaciones e importaciones totales ( $X$ y $M$, respectivamente) se expresan en dólares constantes y tienen una periodicidad mensual. ${ }^{3}$ Las exportaciones están desagregadas en maquila $\left(X^{\{Q \zeta}\right)$, derivados del petróleo $\left(X^{(O)}\right)$ y el resto $\left(X^{(-Q O)}\right)$. Las importaciones están subdivididas

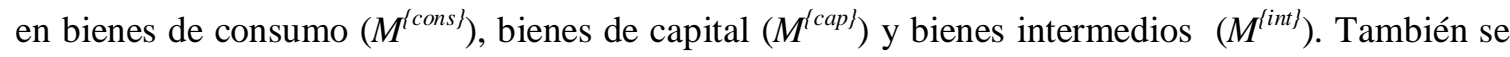
consideran las importaciones de bienes intermedios para maquila $\left(M^{\text {(int } Q l}\right)$ y el resto $\left(M^{\text {(int-Ql}}\right)$. El tipo de cambio real bilateral peso/dólar (TCR) está basado en los respectivos índices de precios al consumidor. Las variables de escala son el índice de valor de la producción industrial de los Estados Unidos (IVUSA) y el índice de valor de la producción industrial de México (IVPI). Los nombres de las variables en letras pequeñas indican el uso de logaritmos.

En el Cuadro 1 se presentan dos pruebas (con tres y seis rezagos) para clasificar los logaritmos de las series como de tendencia estacionaria (TS) o diferencia estacionaria (DS). La primera es la prueba DFGLS propuesta por Elliot et al. (1996), la cual es una versión más poderosa de la bien conocida prueba ADF. La segunda es la prueba KPSS de estacionariedad propuesta por

\footnotetext{
${ }^{3}$ Las importaciones y exportaciones son reportadas cada mes en dólares corrientes. Para los cálculos del documento, estos números se dividen entre el índice de precios al consumidor de los Estados Unidos. De 1980 a 1990 las exportaciones de maquila eran tratadas de manera diferente a las del resto de las exportaciones y eran reportadas como una categoría separada. Desde 1991 las exportaciones de maquila se consideran parte de las exportaciones totales y son también reportadas de modo separado, pero los datos han sido elaborados con una metodología diferente.
} 
Kwiatkowski et al. (1992). Estas pruebas, u otras similares, se usan conjuntamente en análisis confirmatorios de la presencia de una raíz unitaria en las series. ${ }^{4}$

\section{Cuadro 1}

Pruebas de Raíces Unitarias

\begin{tabular}{|c|c|c|c|c|}
\hline \multirow[t]{2}{*}{ VARIABLE } & \multicolumn{2}{|c|}{$D F G L S$} & \multicolumn{2}{|c|}{ PSS2 } \\
\hline & 3 rezagos & 6 rezagos & 3 rezagos & 6 rezagos \\
\hline \multicolumn{5}{|c|}{ NIVELES } \\
\hline $\mathrm{x}$ & -1.8 & -0.9 & $1.3 * *$ & $0.8 * *$ \\
\hline$x^{\{-Q P\}}$ & -1.7 & -1.3 & $0.5 * *$ & $0.3 * *$ \\
\hline $\mathrm{X}^{\{\mathrm{Q}\}}$ & $-2.9 * *$ & -2.2 & $0.2 * *$ & $0.2 * *$ \\
\hline $\mathrm{m}$ & -1.5 & -1.8 & $0.7 * *$ & $0.4^{* *}$ \\
\hline $\mathrm{m}^{\{\mathrm{cap}\}}$ & -1.5 & -1.5 & $0.7 * *$ & $0.4 * *$ \\
\hline $\mathrm{m}^{\{\text {cons }\}}$ & -1.8 & -2.0 & $0.6 * *$ & $0.3^{* *}$ \\
\hline $\mathrm{m}^{\{\text {int }\}}$ & -1.7 & -1.6 & $0.7 * *$ & $0.4^{* *}$ \\
\hline $\mathrm{m}^{\{\text {intQ }\}}$ & $-3.2 * *$ & -2.5 & $0.2 * *$ & 0.1 \\
\hline $\mathrm{m}^{\{\text {int }-\mathrm{Q}\}}$ & -1.7 & -1.7 & $0.7 * *$ & $0.4^{* *}$ \\
\hline ivusa & -1.1 & -1.4 & $0.7 * *$ & $0.4 * *$ \\
\hline ivpi & -1.8 & -1.7 & $0.8 * *$ & $0.5 * *$ \\
\hline tcr & -1.6 & -1.9 & $0.5 * *$ & $0.3 * *$ \\
\hline \multicolumn{5}{|c|}{ DIFERENCIAS } \\
\hline $\mathrm{x}$ & $-8.0 * * *$ & $-3.7 * * *$ & 0.0 & 0.0 \\
\hline$x^{\{-Q P\}}$ & $-8.1 * * *$ & $-3.7 * *$ & 0.0 & 0.0 \\
\hline$x^{\{Q\}}$ & $-10.6 * * *$ & $-6.2 * * *$ & 0.0 & 0.0 \\
\hline $\mathrm{m}$ & $-7.8 * * *$ & $-5.4 * * *$ & 0.1 & 0.1 \\
\hline $\mathrm{m}^{\{\mathrm{cap}\}}$ & $-5.2 * * *$ & $-2.8^{*}$ & 0.0 & 0.1 \\
\hline $\mathrm{m}^{\{\text {cons }\}}$ & $-6.8 * * *$ & $-4.2 * * *$ & 0.1 & 0.1 \\
\hline $\mathrm{m}^{\{\mathrm{int}\}}$ & $-6.0 * * *$ & $-3.7 * * *$ & 0.0 & 0.0 \\
\hline $\mathrm{m}^{\{\text {int } \mathrm{Q}\}}$ & $-10.1 * * *$ & $-6.1 * * *$ & 0.0 & 0.0 \\
\hline $\mathrm{m}^{\{\mathrm{int}-\mathrm{Q}\}}$ & $-5.7 * * *$ & $-3.8 * * *$ & 0.0 & 0.0 \\
\hline ivusa & $-6.7 * * *$ & $-5.1 * * *$ & 0.1 & 0.1 \\
\hline ivpi & $-9.5 * * *$ & $-5.6 * * *$ & 0.0 & 0.0 \\
\hline ter & $-8.3 * * *$ & $-5.5 * * *$ & 0.1 & 0.1 \\
\hline \multicolumn{5}{|c|}{ *** $1 \%$ de significancia } \\
\hline \multirow{2}{*}{\multicolumn{5}{|c|}{ * $* 5 \%$ de significancia }} \\
\hline & & & & \\
\hline
\end{tabular}

En todos los casos excepto dos (con tres rezagos), la prueba DFGLS no rechaza la presencia de una raíz unitaria en los niveles de las series. El resultado es confirmado por el rechazo del estadístico KPSS a la hipótesis de estacionariedad en todos excepto uno de los casos (con seis rezagos). Para las diferencias de las series (tasas de crecimiento) los resultados son los opuestos: el estadístico DFGLS rechaza la hipótesis de una raíz unitaria en todos los casos y el KPSS no puede

\footnotetext{
${ }^{4}$ Para la primera prueba la hipótesis nula es la presencia de una raíz unitaria mientras que para la prueba KPSS la nula es la estacionariedad de la serie. Si DFGLS rechaza y KPSS acepta, entonces la serie es clasificada de modo no ambiguo como TS, mientras que si DFGLS acepta y KPSS rechaza la serie es considerada estacionaria. Cuando ambas pruebas aceptan entonces se concluye que no hay suficiente información en la serie para clasificarla.
} 
rechazar la hipótesis de estacionariedad. Por ello, se procede al análisis considerando que todas las series son I(1). Los resultados de este documento se mantienen inalterados aún si el tipo de cambio real se considera una variable $I(0)$.

\section{Análisis del Comercio Exterior Agregado de México}

En la Gráfica 1 se muestra en ambos paneles con una línea sólida a la balanza comercial de México, medida como $x-m$ en lugar de $X-M$. La línea punteada en el panel superior representa la diferencia del logaritmo de los índices de la producción industrial de México (ivpi) y de los Estados Unidos (ivusa), como un indicador del ingreso relativo. La línea punteada en el panel inferior representa la trayectoria del logaritmo del tipo de cambio real (tcr).

\section{Gráfica 1}

\section{La Balanza Comercial de México y sus Determinantes Principales}

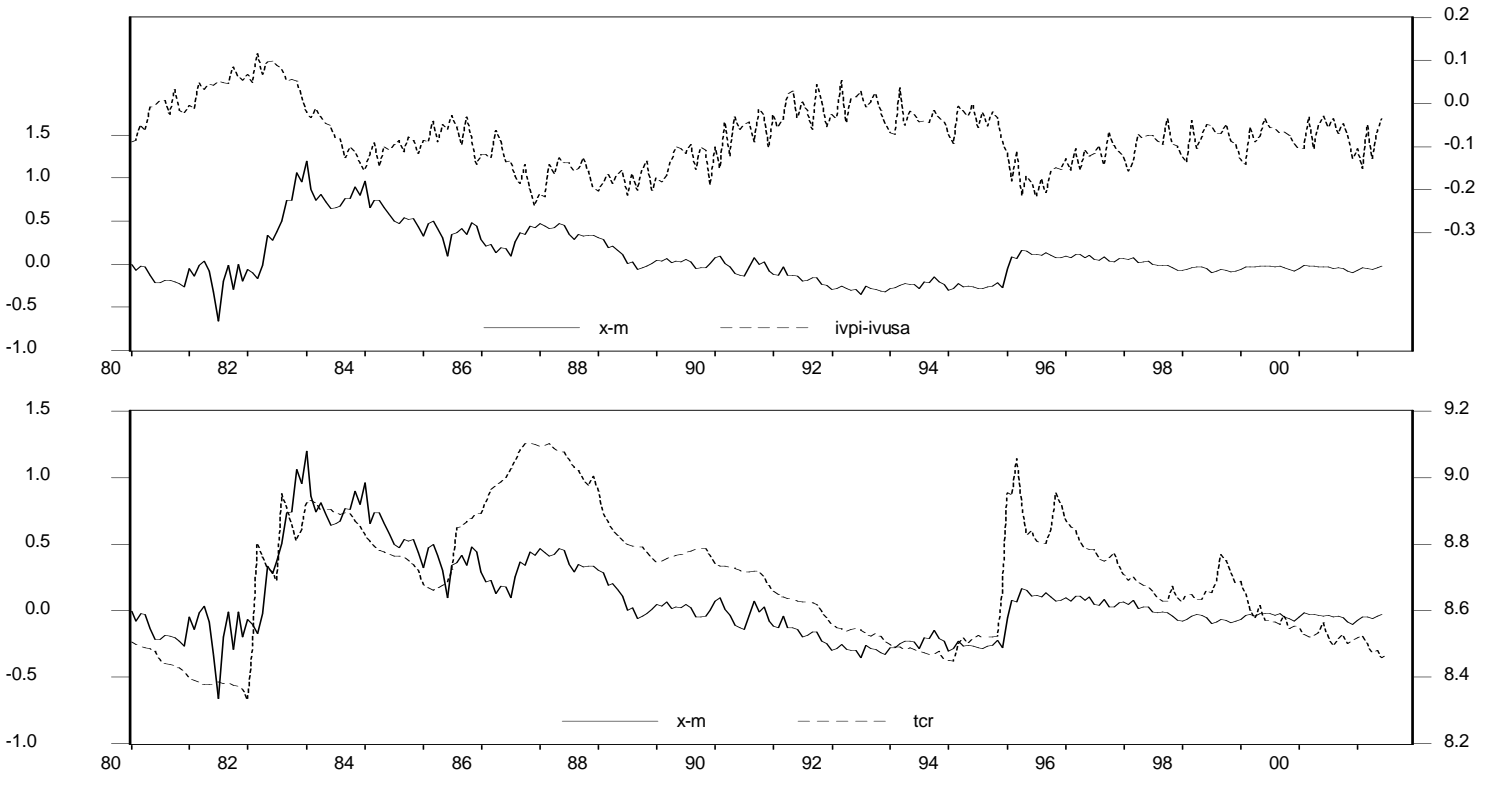

La gráfica sugiere que la balanza comercial mexicana empeora cuando la actividad de México crece más rápido que la de Estados Unidos y lo mismo ocurre cuando el tipo de cambio real se aprecia. El análisis econométrico se basa en considerar un vector $y_{t}=\left(\mathrm{y}_{1 t}, y_{2 t}\right)$ de $k$ variables económicas no estacionarias que forman un sistema cointegrador que puede ser interpretado como una demanda de largo plazo $\mathrm{y}_{1}$ de importaciones o exportaciones, dependiendo de otras $k-1$ variables $y_{2}$ en una forma lineal: 


$$
\mathrm{y}_{1}=\gamma^{\prime} y_{2}
$$

donde $\gamma$ es el $k-1$ vector de coeficientes (elasticidades) de largo plazo. En principio, se puede intentar modelar las ecuaciones para importaciones y exportaciones simultáneamente en el contexto de un Vector de Corrección de Error (VECM). Se aplicó este enfoque pero, debido a la presencia de cambios estructurales (a discutirse después), el tamaño de la muestra disponible es pequeño relativa al número de parámetros y, por ello, es muy sensible a cambios en el número de rezagos. ${ }^{5}$ Por esta razón, se estudia el caso de las exportaciones e importaciones separadamente.

\section{III.1 Exportaciones}

En los cuatro paneles de la Gráfica 2 se observa que desde 1980 ha habido cambios muy importantes en la composición, la tasa de crecimiento y el destino de las exportaciones mexicanas.

\section{Gráfica 2}

\section{Cambios en las Exportaciones Mexicanas}
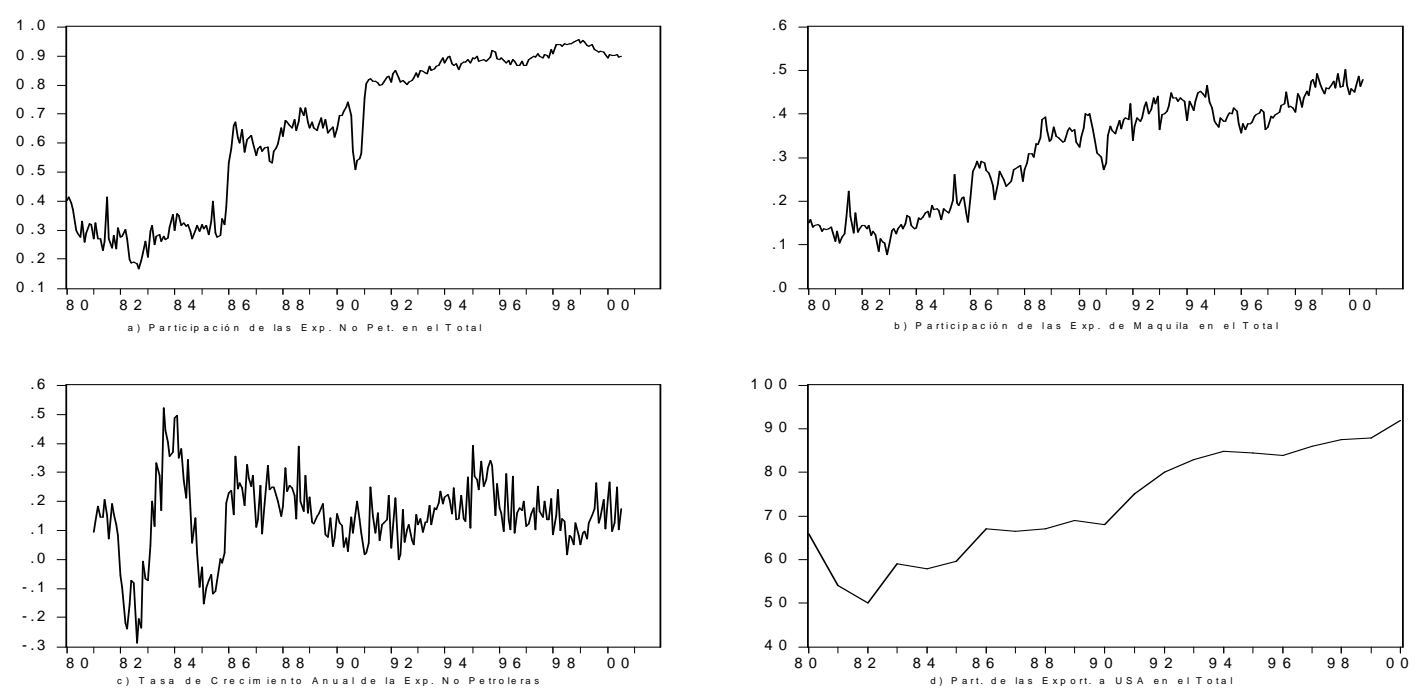

En el panel a) se aprecia que la participación de las exportaciones no petroleras en el total ha aumentado cerca de 50\% al inicio de los ochenta hasta alrededor de $90 \%$ en 2000. En el panel b) se muestra un comportamiento similar de las exportaciones de maquila, las cuales han incrementado su participación en el total hasta alrededor de 50\%, partiendo de 15\% en los inicios de los ochenta.

\footnotetext{
${ }^{5}$ Los coeficientes que se obtienen en este documento pueden ser aproximados por un VECM con nueve rezagos y algunas restricciones que no son aceptadas por los datos.
} 
En el panel c) se ilustra como la evolución de las exportaciones ha cambiado desde la segunda mitad de los ochenta, una vez que México se unió al GATT. Las abruptas variaciones al comienzo de la muestra obedecen a la crisis económica que inició en 1982 y a la aplicación de medidas restrictivas al comercio internacional y son una probable causa de la dificultad para estimar una función de exportaciones para todo el período considerado. En el último panel, que utiliza observaciones anuales por falta de datos mensuales antes de los noventa, se señala que la tendencia de las exportaciones mexicanas a concentrarse hacia los Estados Unidos comenzó desde los ochenta. $^{6}$

En esta sección se modela la demanda de exportaciones mexicanas totales $(x)$ como una función lineal del índice de la producción industrial de los Estados Unidos (ivusa) y del tipo de cambio real (tcr) únicamente para la submuestra de 1990 a 2000 (los diez años previos se estudian más adelante). Se aplica el procedimiento de Johansen (1988) para estudiar el sistema formado por estas tres variables. Los resultados se presentan en el Cuadro 2:

\section{Cuadro 2}

Análisis de Máxima Verosimilitud para Exportaciones 1990-2000

\begin{tabular}{|c|c|c|c|c|}
\hline \multicolumn{5}{|c|}{ (i) Análisis de Cointegración } \\
\hline Eigenvalores & 0.215 & & 0.054 & 0.027 \\
\hline Hipótesis nula & $\operatorname{ran}=0$ & & $\operatorname{ran}<2$ & $\operatorname{ran}<3$ \\
\hline$\lambda_{\text {traza }}$ & $41.280 * *$ & & 10.520 & 3.459 \\
\hline Val. Crit. $(95 \%)$ & 29.700 & & 15.400 & 3.800 \\
\hline \multicolumn{5}{|c|}{ (ii) Estandarización del vector de Cointegración y ajuste de los coeficientes } \\
\hline Variable & $\mathrm{x}$ & & $\mathrm{IPI}^{\{\mathrm{USA}\}}$ & ter \\
\hline Vec. Coin. Nor. $\gamma$ & 1.000 & & -2.800 & -0.318 \\
\hline Ajuste de Coef. $\alpha$ & -0.400 & & 0.017 & 0.043 \\
\hline \multicolumn{5}{|c|}{ (iii) Pruebas de significancia, exogeneidad débil y exogeneidad fuerte de una variable dada } \\
\hline Variable & $\mathrm{x}$ & & $\mathrm{IPI}^{\{\mathrm{USA}\}}$ & $\operatorname{tcr}$ \\
\hline Exclusión $X^{2}(1)$ & $21.247 * *$ & & $19.382 * *$ & $10.656^{* *}$ \\
\hline Exog. débil $X^{2}(1)$ & $16.100 * *$ & & $5.168 *$ & 0.455 \\
\hline \multicolumn{5}{|c|}{ (iv) Pruebas de Especificación } \\
\hline Ecuación & $\mathrm{x}$ & $\operatorname{IPI}^{\{\mathrm{USA}\}}$ & $\operatorname{tcr}$ & Prueba conjunta \\
\hline AR $F(7,102)$ & $4.40[.00] * *$ & $0.75[.63]$ & $1.11[.37]$ & $\mathrm{F}(63,257)=2.01[.00]^{* *}$ \\
\hline Normalidad $X^{2}(2)$ & $0.99[.61]$ & $5.36[.07]$ & $73.98[.00] * *$ & $X^{2}(6)=76.44[.00]^{* *}$ \\
\hline Heteroc. $F(12,96)$ & $1.35[.20]$ & $1.59[.11]$ & $3.08[.00]^{* *}$ & $\mathrm{~F}(72,500)=1.47[.01]^{*}$ \\
\hline \multicolumn{5}{|l|}{ **** $1 \%$ de significancia } \\
\hline \multicolumn{5}{|l|}{ ** $5 \%$ de significnacia } \\
\hline \multicolumn{5}{|l|}{ * $10 \%$ de significancia } \\
\hline \multicolumn{5}{|c|}{ Los valores de probabilidad van entre paréntesis } \\
\hline \multicolumn{5}{|c|}{ Una descripción detallada de las pruebas aquí proporcionadas se pueden obtener en Hendry (1995) } \\
\hline
\end{tabular}

\footnotetext{
${ }^{6}$ De 1940 a 1979 el promedio de la participación de los Estados Unidos en las exportaciones mexicanas fue
} de alrededor de $70 \%$. Antes de los años cincuenta dicha participación llegó a ser mucho mayor. 
La sección (i) contiene los estadísticos de la traza para las hipótesis secuenciales de cero vectores, al menos un vector y a lo más dos vectores de cointegración. Únicamente la primera hipótesis se rechaza y se concluye que hay solamente una relación de largo plazo entre las variables involucradas. Este hecho permite que al darse un coeficiente unitario a las exportaciones $(x)$, los otros coeficientes de la relación sean interpretables como elasticidades de largo plazo.

En el apartado (ii) se presentan los coeficientes de cointegración ya normalizados. Estos tienen el signo correcto y resultan ser muy similares a los que se obtienen más adelante con un método diferente. Los estimados implican la siguiente demanda de largo plazo de las exportaciones totales en dólares constantes $(x)$ :

$$
x=c+2.80 \text { ivusa }+0.32 \text { tcr }
$$

En la sección (iii) del cuadro se incluyen las pruebas de exclusión y exogeneidad débil. Estas demuestran que ninguna de las tres variables puede ser eliminada de la relación de largo plazo y que solamente para las exportaciones totales se rechaza la hipótesis de exogeneidad débil. Lo anterior justifica el análisis más detallado que se lleva a cabo en secciones posteriores utilizando métodos uniecuacionales, aunque una observación es importante en vista de que las pruebas de especificación no fueron completamente satisfactorias.

Si bien se intentó corregir la no normalidad de los residuales del VAR con variables dicotómicas, este proceso requería demasiadas de ellas para el tamaño de muestra. Esto es desafortunado dado que la propiedad de normalidad es un requisito para que las pruebas de esta sección sean estrictamente válidas. Dada esta circunstancia, también presente en la ecuación de las importaciones, se optó por un método de análisis algo diferente para los datos desagregados el cual es más robusto en presencia de no normalidad.

Finalmente, se aplicó una prueba sencilla para evaluar la estabilidad de la función de las exportaciones mexicanas durante el período 1990-2000. Este procedimiento consiste en estimar recursivamente el sistema y probar con un cociente de verosimilitud (distribuido como una $\chi^{2}$ con un grado de libertad) si los coeficientes estimados para cada fecha son estadísticamente iguales a los obtenidos para todo el período. En la Gráfica 3 se presenta la secuencia de cocientes de verosimilitud recursivos junto con la línea que indica el nivel de significancia de 5\%. 
Gráfica 3

Prueba Recursiva de Estabilidad para las Exportaciones

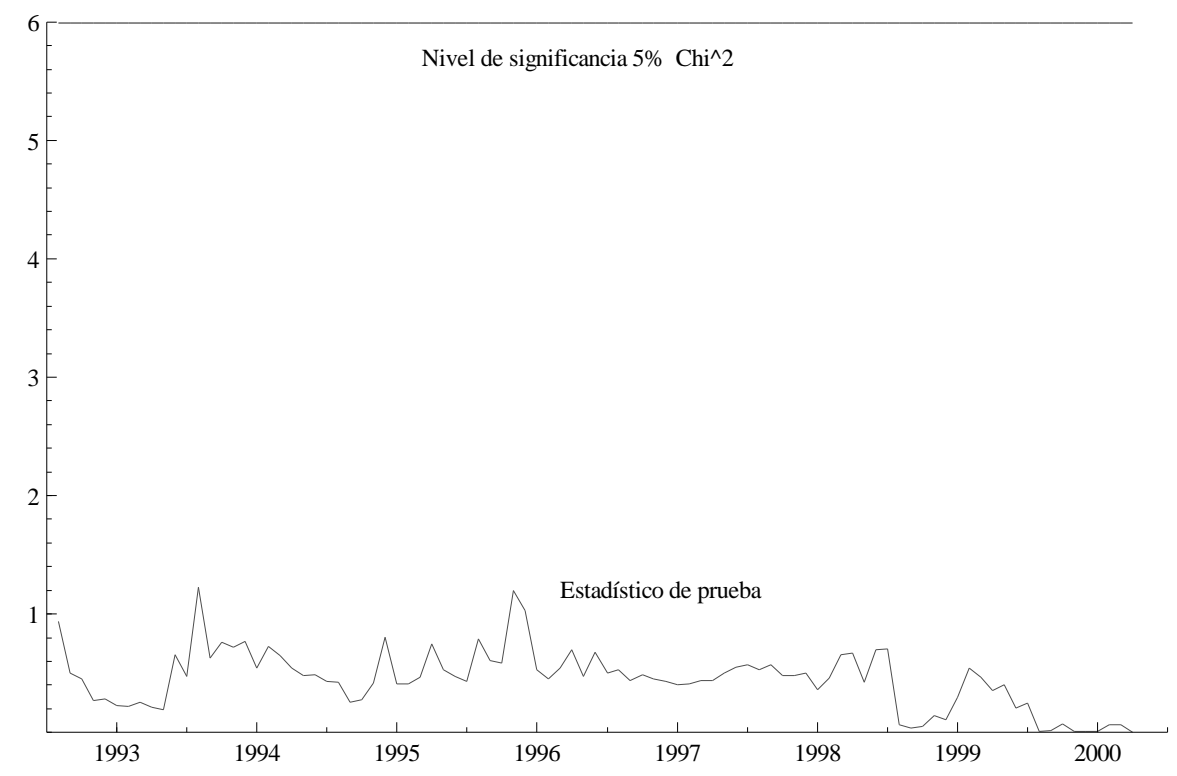

La hipótesis de parámetros constantes es claramente confirmada por los datos. Sobre la base de este resultado y con la salvedad mencionada, puede afirmarse que en la muestra no hay información suficiente para señalar un cambio estructural en la conducta de las exportaciones mexicanas durante los noventa. Sin embargo, este resultado puede ser el producto del nivel de agregación usado en el análisis. Por ello, un análisis con datos más desagregados es necesario. Ejemplo de ello es la trayectoria de las exportaciones petroleras, la cual probablemente no responde a factores de mercado en la misma forma en la que la de otro tipo de exportaciones lo hace. ${ }^{7}$ La precisión del ajuste del modelo se muestra en la Gráfica 4.

\footnotetext{
${ }^{7}$ Por la posible existencia de cuotas de producción.
} 


\section{Gráfica 4}

Las Exportaciones $(x)$ y su Nivel de Largo Plazo $(2.8$ ivusa +0.32 tcr $+c)$

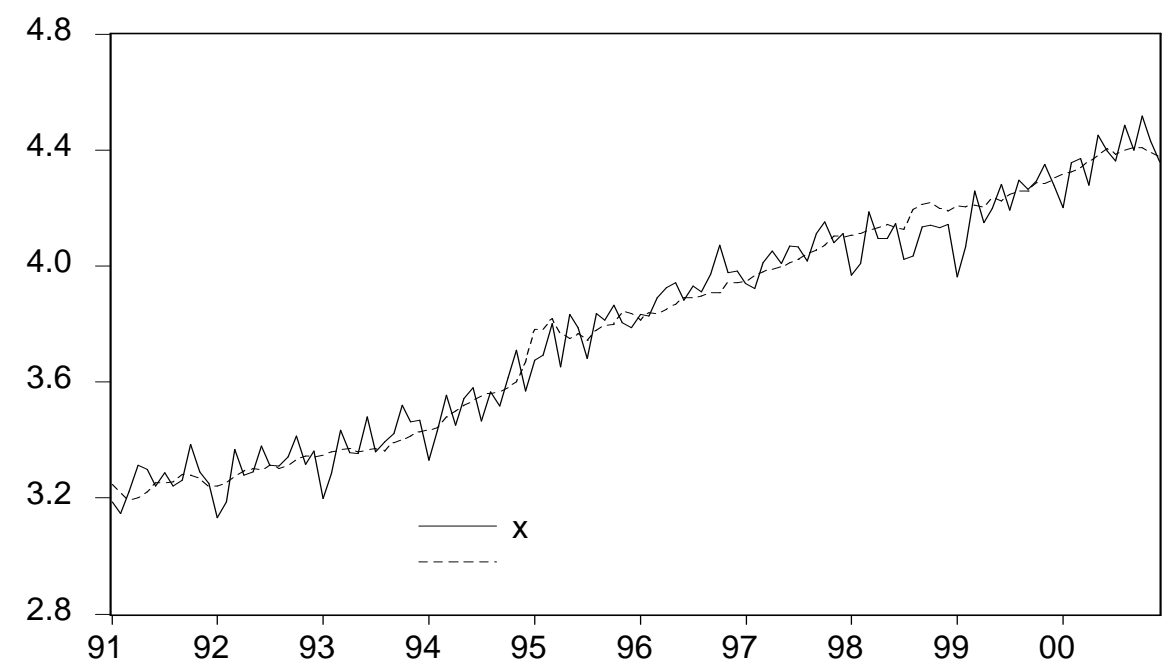

${ }^{8}$ Para este período, la apreciación del tipo de cambio contrarrestó cerca de un tercio del efecto positivo de la actividad en los Estados Unidos 


\section{Gráfica 5}

\section{Evolución de las Importaciones Mexicanas}
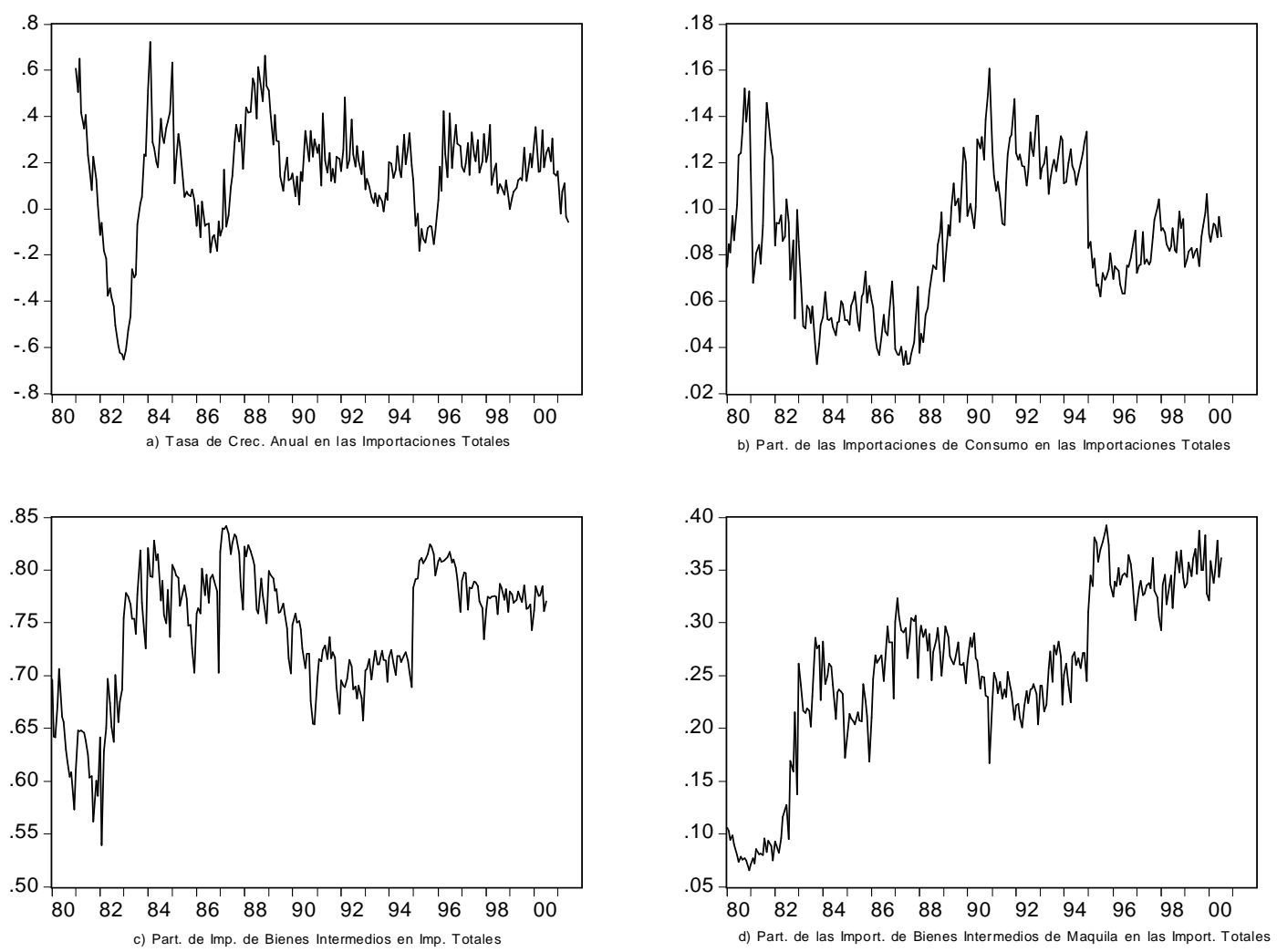

En el panel a) se aprecia la tasa de crecimiento anual de las importaciones totales. Las devaluaciones históricamente han tenido un fuerte impacto sobre dicha variable y algunas veces arribaron junto con severas contracciones de la actividad interna y en el contexto de una política comercial restrictiva (esto último al principio de los ochenta). La fuerte caída que se observa en 1983 ocurrió a consecuencia del estallamiento de la crisis de la deuda.

En el panel b) se presenta la participación de las importaciones de consumo en el total. Este tipo de importaciones tiene el efecto ingreso más elevado. La expansión económica y el aumento del consumo al principio de los noventa coincidió con la etapa en la que las importaciones de este tipo de bienes tuvieron su mayor participación en el total.

En el panel c) se incluye la participación de las importaciones de bienes intermedios en el total. Este es el componente más importante de las importaciones mexicanas y ha estado creciendo desde el comienzo de la muestra, representando actualmente cerca de $80 \%$ del total. En el último 
panel se muestra la participación de los bienes intermedios importados por las maquilas. Este es, por mucho, el componente más vigoroso de las importaciones mexicanas y actualmente se acerca a $30 \%$ del total.

Se aplicó el procedimiento de Johansen a un sistema de variables que incluye importaciones mexicanas totales $(m)$, el índice mexicano de la producción industrial (ivpi), el tipo de cambio real (tcr) y el nivel de las exportaciones totales $(x)$. La muestra considerada en esta sección se extiende de 1991 a 2000. Los resultados se presentan en el Cuadro 3.

\section{Cuadro 3}

Análisis de Máxima Verosimilitud para las Importaciones 1991-2000

\begin{tabular}{|c|c|c|c|c|c|c|}
\hline \multicolumn{7}{|c|}{ (i) Análisis de Cointegración } \\
\hline Eigenvalores & 0.222 & & 0.136 & & 0.029 & 0.002 \\
\hline Hipótesis nula & $\operatorname{ran}=0$ & & $\operatorname{ran}<1$ & & $\operatorname{ran}<2$ & $\operatorname{ran}<3$ \\
\hline$\lambda_{\text {traza }}$ & $48.59 * *$ & & 20.170 & & 3.599 & 0.251 \\
\hline Val. Crit. $(95 \%)$ & 47.200 & & 29.700 & & 15.400 & 3.800 \\
\hline \multicolumn{7}{|c|}{ (ii) Estandarización del vector de Cointegración y ajuste de los coeficientes } \\
\hline Variable & $\mathrm{m}$ & & ivpi & & tcr & $\mathrm{x}$ \\
\hline Vec. Coin. Nor. $\gamma$ & 1.000 & & -0.937 & & 0.410 & -0.530 \\
\hline Ajuste de Coef. $\alpha$ & -0.712 & & -0.178 & & 0.113 & -0.365 \\
\hline \multicolumn{7}{|c|}{ (iii) Pruebas de significancia, exogeneidad débil y exogeneidad fuerte de una variable dada } \\
\hline Variable & $\mathrm{m}$ & & ivpi & & tcr & $\mathrm{x}$ \\
\hline Exclusión $X^{2}(1)$ & $27.98 * *$ & & $24.99 * *$ & & $17.29 * *$ & $26.23 * *$ \\
\hline Exog. débil $X^{2}(1)$ & $16.63 * *$ & & 4.84 & & 1.23 & 4.14 \\
\hline \multicolumn{7}{|c|}{ (iv) Pruebas de Especificación } \\
\hline Ecuación & $\mathrm{m}$ & ivpi & tcr & $x$ & \multicolumn{2}{|c|}{ Prueba conjunta } \\
\hline AR F $(7,97)$ & $2.43[.03]^{*}$ & $2.29[.03]^{*}$ & $2.00[.06]$ & $4.97[.00]^{* *}$ & \multicolumn{2}{|c|}{$\mathrm{F}(112,292)=1.83[.00]^{* *}$} \\
\hline Normalidad $X^{2}(2)$ & $0.12[.94]$ & $1.35[.51]$ & $62.46[.00]^{* *}$ & $0.61[.74]$ & \multicolumn{2}{|c|}{$X^{2}(8)=67.74[.00]^{* *}$} \\
\hline Heteroc. $\mathrm{F}(16,87)$ & $1.10[.37]$ & $1.58[.09]^{*}$ & $3.36[.00]^{* *}$ & $1.84[.04]^{*}$ & \multicolumn{2}{|c|}{$\mathrm{F}(160,685)=1.59[.00]$} \\
\hline \multicolumn{7}{|l|}{$* * * 1 \%$ de significancia } \\
\hline \multicolumn{7}{|l|}{ ** $5 \%$ de significnacia } \\
\hline \multicolumn{7}{|l|}{$* 10 \%$ de significancia } \\
\hline \multicolumn{7}{|c|}{ Los valores de probabilidad van entre paréntesis } \\
\hline \multicolumn{7}{|c|}{ Una descripción detallada de las pruebas aquí proporcionadas se pueden obtener en Hendry (1995) } \\
\hline
\end{tabular}

Estos resultados son muy similares a los que se obtuvieron en el caso de las exportaciones: hay un solo vector de cointegración, las elasticidades de largo plazo tienen el signo correcto, ninguna de las variables puede ser excluida y solamente para la variable importaciones se rechaza la hipótesis de exogeneidad débil. La demanda de largo plazo para las importaciones es la siguiente:

$$
m=c+0.94 i v p i-0.41 t c r+0.53 x
$$

El resultado de las pruebas de estabilidad, sin embargo, es diferente. Como se indica en la Gráfica 6, la secuencia de estadísticos del cociente de verosimilitud cruza la línea de significancia. 


\section{Gráfica 6}

\section{Prueba Recursiva de Estabilidad para las Importaciones}

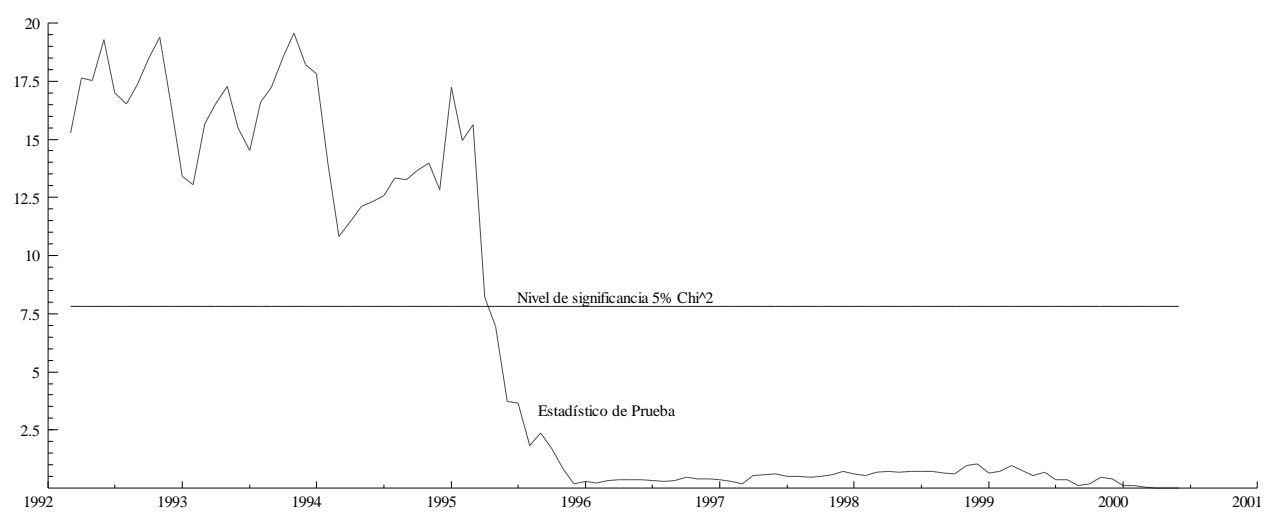

Lo anterior podría ser interpretado como indicativo de que un cambio estructural en las importaciones mexicanas ocurrió durante los noventa. Sin embargo, un análisis más detallado sería necesario. Por ejemplo, el modelo de corrección que se presenta más adelante exhibe estabilidad. Otro argumento contra la hipótesis de cambio estructural en los noventa lo ofrece el hecho de que la ecuación de largo plazo ajusta los datos extremadamente bien, tal como se muestra en la Gráfica 7.

\section{Gráfica 7}

\section{Las Importaciones (m) y su Nivel de Largo Plazo (0.94 ivpi - 0.41 ter + 0.53 x + c)}

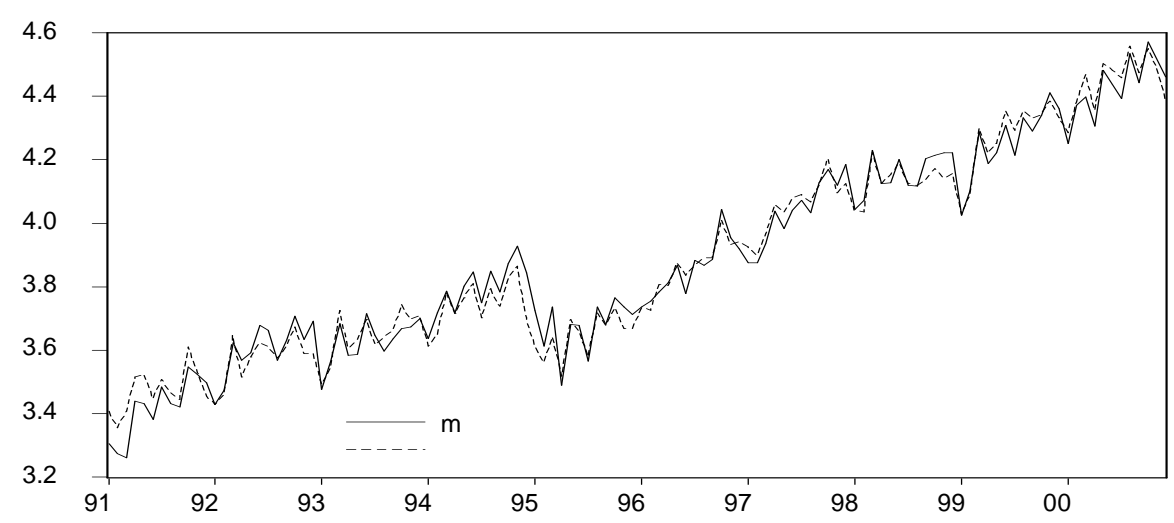


totales podrían no confirmarse a un nivel desagregado. Para considerar esta posibilidad, además de incluir todos los años disponibles en la muestra, se estimó y se examinó la estabilidad de las funciones de exportaciones e importaciones desagregadas para el período completo 1980-2000 y los subperíodos 1980-1989 y 1990-2000. Debido al alto número de funciones estimadas se utilizó el marco del modelo de corrección no restringido, que generalmente produce los mismos resultados que el enfoque de máxima verosimilitud presentado en la sección previa, pero es más simple.

Primero se somete a prueba la propiedad de cointegración para las diferentes definiciones y submuestras y luego se determina la estabilidad de las funciones que tienen tal propiedad. Lo último se lleva a cabo con las pruebas de estabilidad de Hansen (1992) para regresiones con variables I(1) con el método de FM-LS. Los estimados para los datos agregados resultaron muy similares con cada método utilizado y este último es más robusto en cuanto a los problemas de no normalidad comentados antes.

\section{IV.1 Existencia de Relaciones de Largo Plazo con Datos Desagregados}

Se dividió a las exportaciones en maquila $\left(x^{(Q)}\right)$, petroleras $\left(x^{(O)}\right)$ y el resto $\left(x^{(-Q O)}\right)$. Las importaciones se desagregaron en bienes de consumo $\left(m^{(c)}\right)$, bienes de capital $\left(m^{(k)}\right)$ y bienes intermedios $\left(m^{(i)}\right)$. También se consideraron las importaciones de bienes intermedios para maquila $\left(m^{\{i q\}}\right)$ y el resto $\left(m^{(i-q)}\right)$. Para cada categoría se estimaron modelos de corrección de error no restringidos para cada submuestra.

En el Cuadro 4 se reportan solamente los casos donde había evidencia de cointegración para los diferentes tipos de exportaciones. Debido a que los estimados de los vectores de cointegración son muy similares con cada uno de los métodos aplicados, no se reportan las ecuaciones correspondientes. En la Columna 3 se proporciona el número de variables en la relación de cointegración $k$. La columna 4 contiene el número de componentes determinísticos $d$. La columna 5 indica el tamaño de la muestra $T$ y la columna 6 el número total de regresores h. La columna 7 corresponde el estadístico ECM. ${ }^{9}$ La columna final proporciona el valor $p$ para cada estadístico generado por el programa de Ericsson y McKinnon (1999). Cada caso es interesante en sí mismo.

\footnotetext{
${ }^{9}$ El cual es el estadístico del nivel de variables dependientes rezagadas.
} 


\section{Cuadro 4}

Pruebas del MCE para las Exportaciones

\begin{tabular}{|c|c|c|c|c|c|c|c|}
\hline Variable & Periodo & $\begin{array}{c}\text { No. de Variables } \\
\text { en el Vec. Coint. } \\
\mathrm{k}\end{array}$ & $\begin{array}{c}\text { No. De Variables } \\
\text { Deterministicas. } \\
\mathrm{d}\end{array}$ & $\begin{array}{c}\text { Tamaño de } \\
\text { Muestra } \\
\mathrm{T}\end{array}$ & $\begin{array}{c}\text { No. Total de } \\
\text { Regresores } \\
\mathrm{h}\end{array}$ & $\begin{array}{c}\text { Estadístico } \\
\text { MCE } \\
\mathrm{t}\end{array}$ & $\begin{array}{c}\text { valor } \mathrm{p}^{*} \\
\mathrm{p}\end{array}$ \\
\hline \hline $\mathrm{x}$ & $90-2000$ & 3 & 1 & 127 & 7 & -6.38 & 0.00 \\
\hline \multirow{3}{*}{$\mathrm{x}^{\{-\mathrm{QP}\}}$} & $80-2000$ & 3 & 1 & 234 & 7 & -3.67 & 0.04 \\
& $80-89$ & 3 & 1 & 107 & 7 & -3.38 & 0.07 \\
& $91-2000$ & 3 & 1 & 114 & 7 & -4.80 & 0.00 \\
\hline \multirow{3}{*}{$\mathrm{x}^{\{\mathrm{Q}\}}$} & $80-2000$ & 2 & 1 & 234 & 6 & -2.72 & 0.15 \\
& $80-89$ & 2 & 1 & 107 & 6 & -3.90 & 0.01 \\
& $91-2000$ & 2 & 1 & 113 & 6 & -4.88 & 0.00 \\
\hline \hline
\end{tabular}

*Los valores críticos y los valores p fueron obtenidos usando el método de Ericsson y Mackinnon (1999), como implemento en el programa ECMtest.xls (version 1.0).

Para las importaciones totales $(x)$ solamente se encontró cointegración para el período 19902000 (valor $p=0.0$ ) y no para la muestra completa y la razón se puede inferir del análisis de los datos desagregados. El caso de las exportaciones de maquila $\left(x^{(Q)}\right)$ puede explicar la situación. Primero que todo, la relación de cointegración excluye al tipo de cambio real (por ello $k=2$ ). Segundo, para toda la muestra el estadístico ECM no es significativo $(-2.72$ con valor $p=0.15)$. Sin embargo, para cada submuestra, el estadístico ECM lo es. Este es un caso probable de cambio estructural (como se verá, el vector de cointegración es muy diferente para cada submuestra) del tipo sugerido por Gregory y Hansen (1996), donde un cambio estructural esconde la existencia de una relación de largo plazo entre las variables $I(1) .^{\mathbf{1 0}}$

Estas características de las exportaciones de maquila explican el problema de encontrar cointegración para las exportaciones totales. El caso de las exportaciones no petroleras y de no maquila $\left(x^{(-Q O)}\right)$ muestra cointegración para los tres períodos y las variables cointegrantes son las mismas que las relativas a las exportaciones totales.

En el Cuadro 5 se muestra que en importaciones totales hay cointegración solamente para el período 1990-2000. Para esta categoría, las variables cointegrantes son el índice de la producción industrial, el tipo de cambio real y las exportaciones $(k=4)$. Las mismas variables cointegrantes aparecen en el caso de las importaciones de bienes intermedios, pero las exportaciones no están en las ecuaciones de importaciones de bienes de capital y bienes de consumo $(k=3)$.

\footnotetext{
${ }^{10}$ Se aplicaron las pruebas y los resultados, disponibles directamente del autor, fueron coincidentes con los que aquí se presentan. Fue interesante observar que la prueba ADF* tenía demasiada tendencia a rechazar la hipótesis de cointegración. Las otras dos pruebas se desempeñaron más de acuerdo con los resultados reportados de este trabajo.
} 


\section{Cuadro 5}

\section{Pruebas del MCE para las Importaciones}

\begin{tabular}{|c|c|c|c|c|c|c|c|}
\hline Variable & Periodo & $\begin{array}{c}\text { No. de Variables } \\
\text { en el Vec. Coint. } \\
\text { k }\end{array}$ & $\begin{array}{c}\text { No. De Variables } \\
\text { Deterministicas. } \\
\text { d }\end{array}$ & $\begin{array}{c}\text { Tamaño de } \\
\text { Muestra } \\
\mathrm{T} \\
\end{array}$ & $\begin{array}{c}\text { No. Total de } \\
\text { Regresores } \\
\text { h }\end{array}$ & $\begin{array}{c}\text { Estadístico } \\
\text { MCE } \\
t\end{array}$ & $\begin{array}{c}\text { valor } \mathrm{p}^{*} \\
\mathrm{p}\end{array}$ \\
\hline $\mathrm{m}$ & $91-2000$ & 4 & 1 & 113 & 8 & -5.46 & 0.00 \\
\hline \multirow{3}{*}{$\mathrm{m}^{\{\mathrm{cap}\}}$} & $80-2000$ & 3 & 1 & 243 & 6 & -6.00 & 0.00 \\
\hline & 80-89 & 2 & 1 & 116 & 6 & -4.02 & 0.01 \\
\hline & $91-2000$ & 3 & 1 & 114 & 6 & -6.85 & 0.00 \\
\hline \multirow{3}{*}{$\mathrm{m}^{\{\text {cons }\}}$} & $80-2000$ & 3 & 1 & 234 & 8 & -3.93 & 0.02 \\
\hline & $80-89$ & 3 & 1 & 107 & 8 & -3.71 & 0.03 \\
\hline & $91-2000$ & 3 & 1 & 114 & 8 & -5.20 & 0.00 \\
\hline$m^{\{\text {int\} }}$ & $91-2000$ & 3 & 1 & 113 & 6 & -6.37 & 0.00 \\
\hline $\mathrm{m}^{\text {\{intQ\} }}$ & $91-2000$ & 3 & 1 & 113 & 9 & -4.81 & 0.00 \\
\hline \multirow[t]{2}{*}{$m^{\{\text {int }-Q\}}$} & $80-2000$ & 3 & 1 & 245 & 7 & -3.38 & 0.07 \\
\hline & $91-2000$ & 3 & 1 & 113 & 7 & -6.82 & 0.00 \\
\hline
\end{tabular}

El caso de las importaciones de bienes de capital $\left(m^{\{c a p\}}\right)$ es notable porque la presencia de cointegración para toda la muestra esconde la existencia de un cambio estructural de un tipo especial. Se encuentra cointegración en los tres períodos, pero los vectores de las dos submuestras contienen un número diferente de componentes: $k=3$ para el período 1990-2000 y $k=2$ para el período 1980-89. En lo anterior, la variable que sale de la relación de cointegración es el índice de la producción industrial de México. Esta situación de cambio estructural, sin embargo, fue detectada por las pruebas de estabilidad que se reportan más adelante. Para las importaciones de consumo $\left(m^{\{c o n s\}}\right)$ se encontró cointegración para los tres períodos y el mismo número de variables aparece en cada caso. Sin embargo, como se verá, también hay evidencia de un cambio estructural.

Para bienes intermedios $\left(m^{\text {(int) }}\right)$ solamente se encontró cointegración para el período 19912000. Como éste es por mucho el componente más grande de las importaciones mexicanas, la falta de cointegración se pasa al total. Dividiendo esta categoría entre aquéllos bienes que van a maquilas $\left(m^{\text {(int } Q\}}\right)$ y el resto $\left(m^{\text {(int- } Q\}}\right)$ se llega a resultados similares.

\section{IV.2 Estabilidad de las Relaciones de Largo plazo con Datos Desagregados}

En esta sección se examina la estabilidad de las relaciones de largo plazo encontradas antes. Para los mismos casos presentados en el Cuadro 4, se estimaron coeficientes de cointegración con el método de cuadrados mínimos completamente modificados (FM-LS por sus siglas en inglés) y se aplicaron tres pruebas de estabilidad para variables I(1) propuestas por Hansen (1991). Las tres tienen como hipótesis nula coeficientes de cointegración constantes durante toda la muestra. Las 
primeras dos (LC y F prom) tienen a una caminata aleatoria como alternativa mientras que la tercera (F sup) tiene a un cambio de una sola vez en una fecha desconocida. ${ }^{11}$

Las columnas 3 a 5 del Cuadro 6 contienen los estimados FM-LS de los coeficientes de largo plazo de las funciones de exportaciones junto con los errores estándar para cada una de las variables cointegrantes y una constante. En las columnas 6 a 8 se presenta el valor de los estadísticos para probar estabilidad y los respectivos valores $p .^{12}$

\section{Cuadro 6}

Estabilidad de la Relación de Largo Plazo para las Exportaciones

\begin{tabular}{|c|c|c|c|c|c|c|c|}
\hline \multirow[t]{2}{*}{ Variable } & \multirow[t]{2}{*}{ Periodo } & \multicolumn{3}{|c|}{ Relaciones de Largo Plazo* } & \multicolumn{3}{|c|}{ Pruebas de Estabilidad** } \\
\hline & & $\mathrm{IPI}^{\mathrm{USA}}$ & tcr & $\mathrm{C}$ & $\mathrm{LC}$ & F prom. & F sup. \\
\hline $\mathrm{x}$ & $90-2000$ & $\begin{array}{c}2.867 \\
(0.070)\end{array}$ & $\begin{array}{c}0.234 \\
(0.070)\end{array}$ & $\begin{array}{r}-11.890 \\
(0.656)\end{array}$ & $\begin{array}{c}0.321 \\
\mathrm{p}=0.200\end{array}$ & $\begin{array}{c}5.090 \\
p=0.107\end{array}$ & $\begin{array}{c}7.680 \\
p=0.200\end{array}$ \\
\hline \multirow{3}{*}{$x^{\{-Q P\}}$} & $80-2000$ & $\begin{array}{c}3.597 \\
(0.105) \\
\end{array}$ & $\begin{array}{c}0.069 \\
(0.100) \\
\end{array}$ & $\begin{array}{r}-14.756 \\
(1.046) \\
\end{array}$ & $\begin{array}{c}0.884 \\
\mathrm{p}=0.010\end{array}$ & $\begin{array}{c}10.759 \\
\mathrm{p}=0.010\end{array}$ & $\begin{array}{c}20.371 \\
\mathrm{p}=0.010\end{array}$ \\
\hline & $80-89$ & $\begin{array}{c}2.917 \\
(0.302)\end{array}$ & $\begin{array}{c}0.163 \\
(0.123) \\
\end{array}$ & $\begin{array}{r}-12.595 \\
(1.351) \\
\end{array}$ & $\begin{array}{c}0.479 \\
\mathrm{p}=0.111\end{array}$ & $\begin{array}{c}5.382 \\
\mathrm{p}=0.088\end{array}$ & $\begin{array}{c}13.884 \\
p=0.082\end{array}$ \\
\hline & $91-2000$ & $\begin{array}{c}2.945 \\
(0.0712\end{array}$ & $\begin{array}{c}0.702 \\
(0.067)\end{array}$ & $\begin{array}{r}-17.077 \\
(0.608)\end{array}$ & $\begin{array}{c}0.258 \\
\mathrm{p}=0.200\end{array}$ & $\begin{array}{c}5.003 \\
p=0.114\end{array}$ & $\begin{array}{c}10.460 \\
p=0.200\end{array}$ \\
\hline \multirow[t]{2}{*}{$x^{\{Q\}}$} & $80-89$ & $\begin{array}{c}4.564 \\
(0.199)\end{array}$ & . & $\begin{array}{r}-18.974 \\
(0.889)\end{array}$ & $\begin{array}{c}0.772 \\
\mathrm{p}=0.018\end{array}$ & $\begin{array}{c}7.560 \\
p=0.010\end{array}$ & $\begin{array}{c}12.105 \\
\mathrm{p}=0.055\end{array}$ \\
\hline & $91-2000$ & $\begin{array}{c}3.279 \\
(0.074)\end{array}$ & . & $\begin{array}{r}-12.706 \\
(0.353)\end{array}$ & $\begin{array}{c}0.176 \\
p=0.200\end{array}$ & $\begin{array}{c}1.831 \\
\mathrm{p}=0.200\end{array}$ & $\begin{array}{c}4.897 \\
p=0.200\end{array}$ \\
\hline
\end{tabular}

Lo primero a notar es que las elasticidades para las exportaciones totales calculadas con el método FM-LS son muy similares a aquéllas obtenidas con el método de Johansen y a las conseguidas con el modelo de corrección de error no restringido. Las pruebas de estabilidad confirman los resultados comentados en la sección III, que no mostraban evidencia de inestabilidad en la relación de largo plazo: el valor $p$ de cada prueba estadística se encuentra arriba de 0.20.

Para las exportaciones no petroleras y sin maquila $\left(x^{\{-Q P)}\right)$ se encontró una relación de largo plazo para los tres períodos. Sin embargo, para la muestra completa los estadísticos de prueba detectan inestabilidad. Para cada una de las dos submuestras los vectores de cointegración difieren principalmente en cuanto al coeficiente del tipo de cambio real. En este caso parece haber ocurrido un cambio estructural, pero no tan drástico como para encubrir la presencia de una relación de largo plazo. Las exportaciones de maquila ejemplifican una situación donde el cambio en los coeficientes

\footnotetext{
${ }^{11}$ Ver Hansen (1991) para una descripción completa de estas pruebas.

12 Todos los cálculos numéricos de esta sección fueron generados con el programa en GAUSS obtenible del sitio de Internet de Bruce Hansen.
} 
esconde la existencia de una relación de cointegración. Para toda la muestra no fue posible encontrar algún vector razonable, pero para cada submuestra sí. ${ }^{13}$ El tipo de cambio real no es significativo en ningún vector, y la elasticidad del índice de la producción industrial de los Estados Unidos es mayor en la primera submuestra donde, además, los estadísticos detectan probable inestabilidad. Este problema no aparece en la segunda submuestra.

En el cuadro 7 se presentan los resultados correspondientes a las importaciones. La interpretación es la misma que en el Cuadro 6.

\section{Cuadro 7}

Estabilidad de la Relación de Largo Plazo para las Importaciones

\begin{tabular}{|c|c|c|c|c|c|c|c|c|}
\hline \multirow[t]{2}{*}{ Variable } & \multirow[t]{2}{*}{ Periodo } & \multicolumn{4}{|c|}{ Relaciones de Largo Plazo* } & \multicolumn{3}{|c|}{ Pruebas de Estabilidad** } \\
\hline & & $\mathrm{IPI}^{\mathrm{USA}}$ & ter & $\mathrm{x}$ & $\mathrm{C}$ & LC & F prom. & F sup. \\
\hline$\overline{\mathrm{m}}$ & 91-2000 & $\begin{array}{c}1.124 \\
(0.131)\end{array}$ & $\begin{array}{c}-0.286 \\
(0.061)\end{array}$ & $\begin{array}{c}0.461 \\
(0.043)\end{array}$ & $\begin{array}{c}-0.676 \\
(0.922)\end{array}$ & $\begin{array}{c}0.863 \\
p=0.020\end{array}$ & $\begin{array}{c}12.054 \\
\mathrm{p}=0.010\end{array}$ & $\begin{array}{c}24.897 \\
p=0.010\end{array}$ \\
\hline \multirow{3}{*}{$\mathrm{m}^{\{\text {cap }\}}$} & $80-2000$ & $\begin{array}{c}2.217 \\
(0.151)\end{array}$ & $\begin{array}{c}-1.570 \\
(0.141)\end{array}$ & . & $\begin{array}{c}5.141 \\
(1.623) \\
\end{array}$ & $\begin{array}{c}1.518 \\
\mathrm{p}=0.010\end{array}$ & $\begin{array}{c}9.624 \\
p=0.010\end{array}$ & $\begin{array}{c}22.547 \\
p=0.010\end{array}$ \\
\hline & $80-89$ & . & $\begin{array}{l}-1.966 \\
(0.237)\end{array}$ & . & $\begin{array}{l}18.300 \\
(2.082)\end{array}$ & $\begin{array}{c}0.762 \\
\mathrm{p}=0.019\end{array}$ & $\begin{array}{c}5.561 \\
p=0.025\end{array}$ & $\begin{array}{c}11.455 \\
\mathrm{p}=0.071\end{array}$ \\
\hline & $91-2000$ & $\begin{array}{c}1.966 \\
(0.077)\end{array}$ & $\begin{array}{c}-0.734 \\
(0.068)\end{array}$ & . & $\begin{array}{c}-0.927 \\
(0.728)\end{array}$ & $\begin{array}{c}0.173 \\
\mathrm{p}=0.200\end{array}$ & $\begin{array}{c}2.658 \\
\mathrm{p}=0.200\end{array}$ & $\begin{array}{c}9.215 \\
\mathrm{p}=0.200\end{array}$ \\
\hline \multirow{3}{*}{$\mathrm{m}^{\{\text {cons }\}}$} & $80-2000$ & $\begin{array}{c}3.506 \\
(0.376) \\
\end{array}$ & $\begin{array}{c}-2.277 \\
(0.350) \\
\end{array}$ & . & $\begin{array}{c}4.720 \\
(4.039) \\
\end{array}$ & $\begin{array}{c}0.962 \\
p=0.010\end{array}$ & $\begin{array}{c}9.488 \\
p=0.010\end{array}$ & $\begin{array}{c}17.121 \\
p=0.023\end{array}$ \\
\hline & $80-89$ & $\begin{array}{c}5.324 \\
(1.571)\end{array}$ & $\begin{array}{c}-2.188 \\
(0.402)\end{array}$ & . & $\begin{array}{l}-4.095 \\
(8.802)\end{array}$ & $\begin{array}{c}0.696 \\
p=0.035\end{array}$ & $\begin{array}{c}5.818 \\
p=0.066\end{array}$ & $\begin{array}{c}14.974 \\
\mathrm{p}=0.054\end{array}$ \\
\hline & $91-2000$ & $\begin{array}{c}1.753 \\
(0.150) \\
\end{array}$ & $\begin{array}{c}-1.160 \\
(0.132) \\
\end{array}$ & . & $\begin{array}{l}3.30677 \\
(1.411) \\
\end{array}$ & $\begin{array}{c}0.086 \\
\mathrm{p}=0.200\end{array}$ & $\begin{array}{c}1.537 \\
p=0.200\end{array}$ & $\begin{array}{c}7.990 \\
p=0.200\end{array}$ \\
\hline$m^{\{\text {int }\}}$ & $91-2000$ & . & $\begin{array}{c}-0.476 \\
(0.048)\end{array}$ & $\begin{array}{c}0.908 \\
(0.017)\end{array}$ & $\begin{array}{c}4.261 \\
(0.399)\end{array}$ & $\begin{array}{c}0.331 \\
\mathrm{p}=0.200\end{array}$ & $\begin{array}{c}4.806 \\
p=0.129\end{array}$ & $\begin{array}{c}10.862 \\
p=0.200\end{array}$ \\
\hline $\mathrm{m}^{\{\text {int_Q\}}}$ & $91-2000$ & . & $\begin{array}{c}-0.206 \\
(0.071)\end{array}$ & $\begin{array}{c}1.224 \\
(0.026)\end{array}$ & $\begin{array}{c}-0.181 \\
(0.592)\end{array}$ & $\begin{array}{c}0.567 \\
p=0.067\end{array}$ & $\begin{array}{c}10.247 \\
\mathrm{p}=0.010\end{array}$ & $\begin{array}{c}20.327 \\
p=0.010\end{array}$ \\
\hline \multirow[t]{2}{*}{$\mathrm{m}^{\{\text {int_-QP }\}}$} & $80-2000$ & $\begin{array}{c}2.549 \\
(0.502)\end{array}$ & $\begin{array}{l}-0.719 \\
(0.186)\end{array}$ & $\begin{array}{c}0.036 \\
(0.182)\end{array}$ & $\begin{array}{l}-2.833 \\
(2.923)\end{array}$ & $\begin{array}{c}1.388 \\
p=0.010\end{array}$ & $\begin{array}{c}11.626 \\
p=0.010\end{array}$ & $\begin{array}{c}19.623 \\
p=0.264\end{array}$ \\
\hline & $91-2000$ & $\begin{array}{c}1.200 \\
(0.158)\end{array}$ & $\begin{array}{c}-0.146 \\
(0.074)\end{array}$ & $\begin{array}{c}0.323 \\
(0.053)\end{array}$ & $\begin{array}{c}-2.523 \\
(1.118)\end{array}$ & $\begin{array}{c}1.000 \\
\mathrm{p}=0.010\end{array}$ & $\begin{array}{c}10.014 \\
\mathrm{p}=0.010\end{array}$ & $\begin{array}{c}15.524 \\
\mathrm{p}=0.113\end{array}$ \\
\hline
\end{tabular}

Para las importaciones totales solamente se encontró un vector de cointegración para el período 1991- 2000. Este es muy similar al obtenido en la sección III (en particular, el nivel exportaciones es parte del conjunto cointegrador). Para las importaciones de bienes de capital fue posible encontrar un vector de cointegración para cada período. Si bien el obtenido para toda la muestra y la primera submuestra muestra inestabilidad, la segunda submuestra es muy estable. Tanto para este tipo de importaciones, como para las de bienes de consumo, el nivel de

\footnotetext{
${ }^{13}$ Es también factible que lo anterior no refleje necesariamente la existencia de un cambio estructural sino alguna modificación metodológica en la recopilación y elaboración de las cifras.
} 
exportaciones no entra en la relación de largo plazo, lo cual es intuitivamente razonable. La variable de escala (el índice de la producción industrial de México) no entró en la primera submuestra. Esta es una situación donde el cambio estructural no esconde la relación de largo plazo.

El caso de las importaciones de bienes de consumo también indica un vector de cointegración inestable para toda la muestra. Sin embargo, los correspondientes a las dos submuestras son estables, especialmente el de la segunda.

Para las importaciones de bienes intermedios solamente se encontró cointegración para la segunda submuestra. El vector de cointegración fue muy estable y no incluye al índice de la producción industrial pero sí al nivel de las exportaciones. De hecho, desagregando los datos aún más, se encontró que las importaciones para la industria de maquila solamente presentan una relación de cointegración inestable en la segunda submuestra. Para los bienes intermedios importados de la industria no maquiladora se encontró cointegración para ambas submuestras pero no para todo el período. La estabilidad en estos casos no es obvia, puesto que los resultados de las pruebas son contradictorios (una de ellas sugiere estabilidad y las otras dos rechazan esta hipótesis).

Las conclusiones de esta sección pueden sintetizarse como sigue. Primero, para todo el período 1980-2000 no es posible encontrar una relación de cointegración para cada uno de los tipos de importaciones y exportaciones que aquí se analizan. Sin embargo, para la mayor parte de las categorías se encontraron relaciones de largo plazo estables para el período 1990-2000. Las únicas excepciones parecen ser los datos a nivel muy desagregado para las importaciones de bienes intermedios.

Finalmente, en la Gráfica 8 se muestra con una línea sólida el logaritmo de las exportaciones no petroleras y su nivel de largo plazo construido en partes (donde una ecuación diferente ajusta cada segmento de la muestra) y donde no se incorporan ni la dinámica de corto plazo (rezagos) ni factores estacionales. Es notable que con el paso del tiempo el nivel de las exportaciones parece volverse más volátil alrededor de su tendencia de largo plazo. 


\section{Gráfica 8}

Las Exportaciones No Petroleras y su Nivel de Largo Plazo

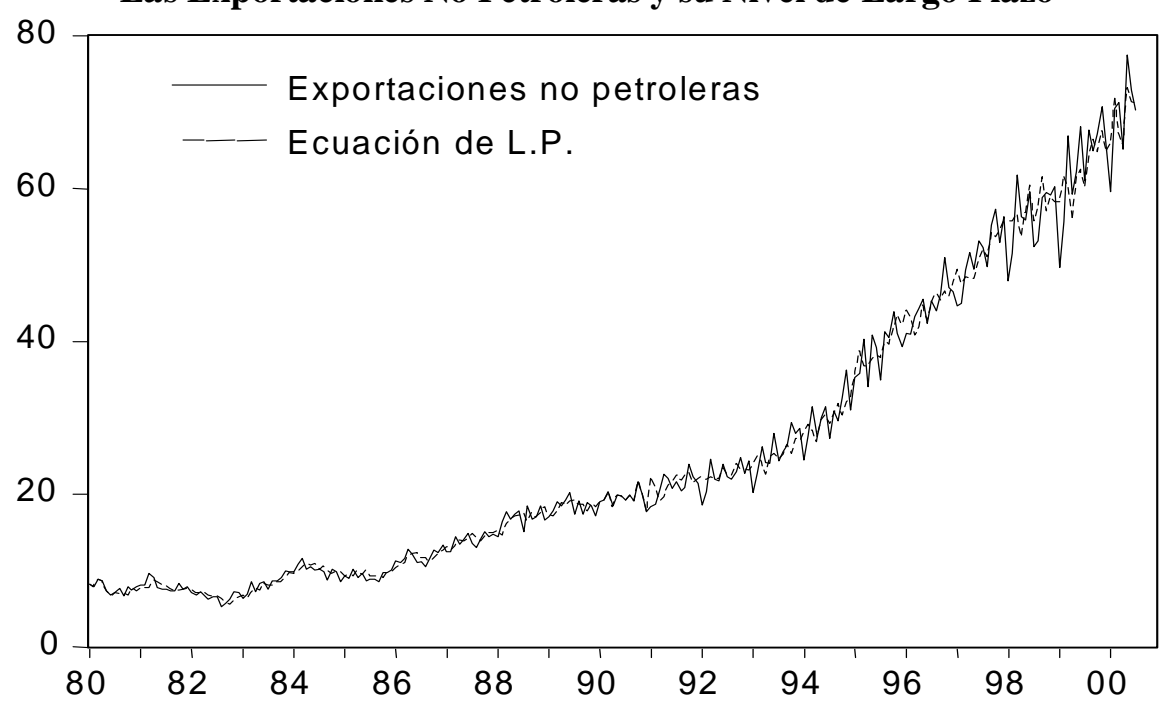

\section{Modelos de Corrección de Error}

En esta sección se analizan las propiedades estadísticas de los modelos de corrección de error asociados a las relaciones de largo plazo para el período 1990-2000. Los términos de corrección de error se construyen con los coeficientes de los Cuadros 6 y 7.

Los cuadros que siguen muestran los estimados de los parámetros de corto plazo y, al final, varios estadísticos de prueba. Una variable con superíndice $x^{i}=x, x^{\{-Q P\}}, x^{\{Q\}}$ indica que ésta debe ser entendida como específica a una ecuación dada. El Cuadro 8 contiene los resultados para las exportaciones. 


\section{Cuadro 8}

Modelos de Corrección de Error para las Exportaciones

\begin{tabular}{|c|c|c|c|}
\hline \multirow[t]{2}{*}{ Variable } & \multicolumn{3}{|c|}{ Modelos } \\
\hline & $\Delta \mathrm{x}$ & $\Delta \mathrm{x}^{\{-\mathrm{QP}\}}$ & $\Delta \mathrm{x}^{\{\mathrm{Q}\}}$ \\
\hline $\operatorname{Cex}_{\mathrm{t}-1}\{\mathrm{i}\}$ & $\begin{array}{c}-0.314 \\
(0.077)\end{array}$ & $\begin{array}{l}-0.271 \\
(0.085)\end{array}$ & $\begin{array}{l}-0.592 \\
(0.123)\end{array}$ \\
\hline$\Delta \mathrm{x}_{\mathrm{t}-1}^{\{\mathrm{i}\}}$ & $\begin{array}{l}-0.420 \\
(0.085)\end{array}$ & $\begin{array}{l}-0.483 \\
(0.081)\end{array}$ & $\begin{array}{l}-0.444 \\
(0.102)\end{array}$ \\
\hline$\Delta \mathrm{x}_{\mathrm{t}-2}^{\{\mathrm{i}\}}$ & $\begin{array}{l}-0.237 \\
(0.078)\end{array}$ & $\begin{array}{l}-0.358 \\
(0.073)\end{array}$ & $\begin{array}{l}-0.303^{\circ o} \\
(0.082)\end{array}$ \\
\hline$\Delta \mathrm{x}^{\{\mathrm{i}\}}{ }_{\mathrm{t}-4}$ & $\begin{array}{l}-0.231 \\
(0.075)\end{array}$ & & \\
\hline$\Delta \mathrm{x}^{\{\mathrm{i}\}}{ }_{\mathrm{t}-5}$ & $\begin{array}{l}-0.294 \\
(0.077)\end{array}$ & & \\
\hline$\Delta \mathrm{x}^{\{\mathrm{i}\}}$ & $\begin{array}{c}0.225 \\
(0.072)\end{array}$ & & \\
\hline$\Delta \mathrm{x}_{\mathrm{t}-10}^{\{\mathrm{i}\}}$ & & & $\begin{array}{c}-0.161 \\
(0.062)\end{array}$ \\
\hline$\Delta \mathrm{x}_{\mathrm{t}-11}^{\{\mathrm{i}\}}$ & & $\begin{array}{c}0.304 \\
(0.075)\end{array}$ & \\
\hline$\Delta x_{t-12}^{\{i\}}$ & $\begin{array}{c}0.245 \\
(0.071)\end{array}$ & $\begin{array}{c}0.358 \\
(0.069)\end{array}$ & $\begin{array}{c}0.214 \\
(0.062)\end{array}$ \\
\hline$\Delta \mathrm{tcr}$ & & $\begin{array}{c}0.417 \\
(0.121)\end{array}$ & \\
\hline$\Delta \mathrm{IPI}^{\{\mathrm{USA}\}}{ }_{\mathrm{t}-5}$ & $\begin{array}{c}2.222 \\
(0.897) \\
\end{array}$ & & \\
\hline Est. MCE & -6.380 & -4.800 & -4.880 \\
\hline $\mathrm{R}^{2}$ & 0.769 & 0.713 & 0.839 \\
\hline $\mathrm{R}^{2}$ Ajust. & 0.728 & 0.679 & 0.812 \\
\hline E.E. & 0.045 & 0.053 & 0.051 \\
\hline Coef. Asimetría & 0.154 & 0.226 & -0.072 \\
\hline Curtosis & 3.472 & 3.583 & 2.656 \\
\hline Est. Jarque-Bera & 1.680 & 2.587 & 0.662 \\
\hline $\mathrm{LM}_{12} \mathrm{AR}$ & 1.387 & 0.728 & 0.771 \\
\hline $\mathrm{LM}_{12} \mathrm{ARCH}$ & $1.970 * *$ & 0.627 & 0.786 \\
\hline Prueba de White & $1.502 *$ & 0.030 & 1.019 \\
\hline RESET & 0.002 & 0.013 & 0.474 \\
\hline Chow B. (94:01) & 1.016 & 0.793 & $1.901 * *$ \\
\hline Chow Pred.( 94:01) & 1.224 & 0.839 & 0.967 \\
\hline
\end{tabular}

$\operatorname{Cex}_{\mathrm{t}-1}=\mathrm{x}_{\mathrm{t}-1-1}-2.867$ IPI $_{\mathrm{t}-1}^{\{\mathrm{USA}\}}-0.234$ tcr $_{\mathrm{t}-1}$

$\mathrm{Cex}_{\mathrm{t}-1}{ }^{\{-\mathrm{QP}\}}=\mathrm{x}_{\mathrm{t}-1}{ }^{\{-\mathrm{QP}\}}-2.945 \mathrm{IPI}^{\{\mathrm{USA}\}}{ }_{\mathrm{t}-1}-0.702 \mathrm{tcr}_{\mathrm{t}-1}$

$\mathrm{Cex}_{\mathrm{t}-1}^{\{\mathrm{Q}\}}=\mathrm{x}_{\mathrm{t}-1}{ }^{\{\mathrm{Q}\}}-3.279 \mathrm{IPI}^{\{\mathrm{USA}\}}{ }_{\mathrm{t}-1}$ 
Los coeficientes de velocidad de ajuste ${ }^{14}$ son todos negativos y muy significativos, fortaleciendo la hipótesis de existencia de una relación de largo plazo. En todos los casos el valor es muy alto, indicando que el proceso de ajuste no toma mucho tiempo para ser completado. Hay varios rezagos de la variable dependiente, principalmente negativos, y eso produce un oscilante proceso de ajuste. En el caso de las exportaciones totales, los coeficientes relativos al cambio en la actividad económica de los Estados Unidos son muy altos. El efecto de corto plazo de variaciones en el tipo de cambio real aparece solamente en la ecuación de las exportaciones sin petróleo y sin maquila.

Todos los estadísticos ECM (obtenidos con el modelo no restringido) son significativos al nivel de 1\%. Las medidas de bondad de ajuste son muy buenas. No hay problemas con la normalidad de los residuales y solamente la ecuación para las exportaciones totales muestra alguna evidencia de efectos ARCH. Las pruebas de Chow de quiebre estructural y falla de pronóstico para enero de 1994 son todas no significativas, con la excepción de un caso. Se introdujo una variable igual a uno al comienzo de 1994 y ésta fue no significativa.

En el Gráfica 9 se presenta el análisis recursivo de los modelos de corrección de error para las exportaciones totales.

\section{Gráfica 9}

Pruebas Recursivas de Estabilidad para los Modelos de Corrección de Error para las Exportaciones Totales.
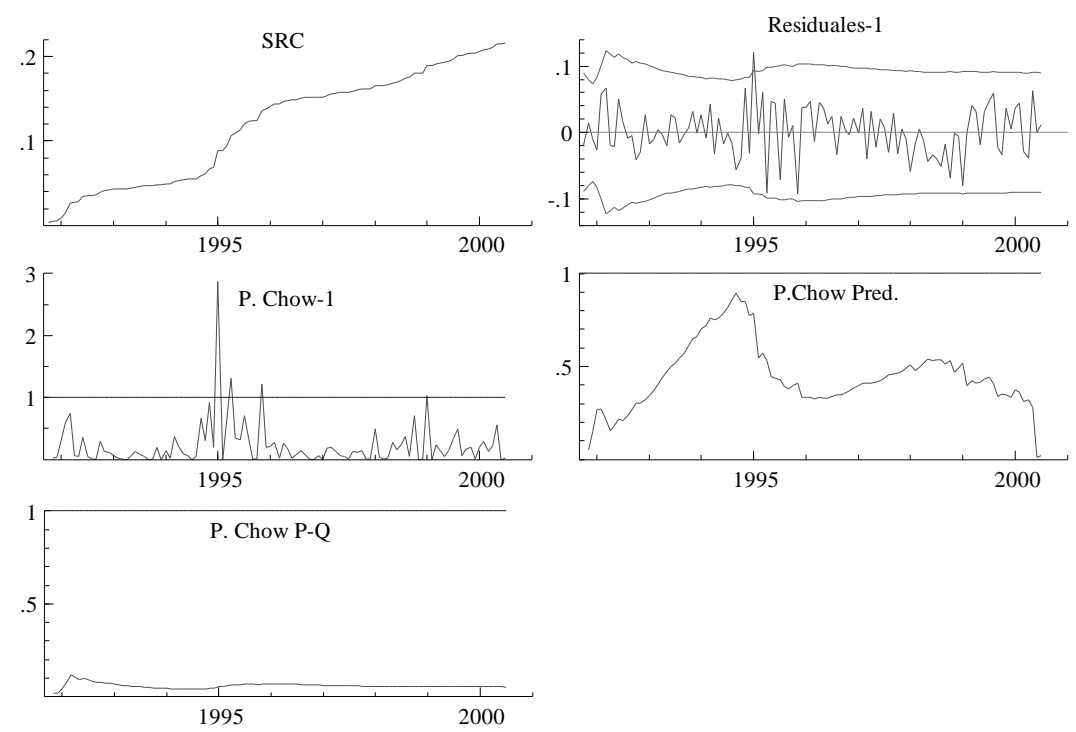

\footnotetext{
${ }^{14}$ Que en el modelo de corrección de error no restringido corresponden al estadístico ECM que se reporta en el mismo cuadro.
} 
El primer panel muestra la suma de cuadrados, la cual se aproxima a una línea recta; el segundo panel muestra la secuencia de residuales de un paso adelante que cruza la banda de confianza solamente en un mes. El siguiente panel muestra los estadísticos de Chow que solamente detectan tres casos de observaciones extremas al tiempo de la crisis de 1995. Los últimos dos paneles muestran los estadísticos de n-pasos hacia adelante y n-pasos hacia atrás de cambio estructural de Chow. En ambos casos la secuencia permanece debajo de la línea de significancia al 5\%. En la Gráfica 10 se muestra la secuencia de coeficientes recursivos para el mismo modelo de corrección de error. Cabe señalar que las secuencias para las "dummies" estacionales también están incluidas.

\section{Gráfica 10}

\section{Coeficientes Recursivos para el Modelo de las Exportaciones Totales}
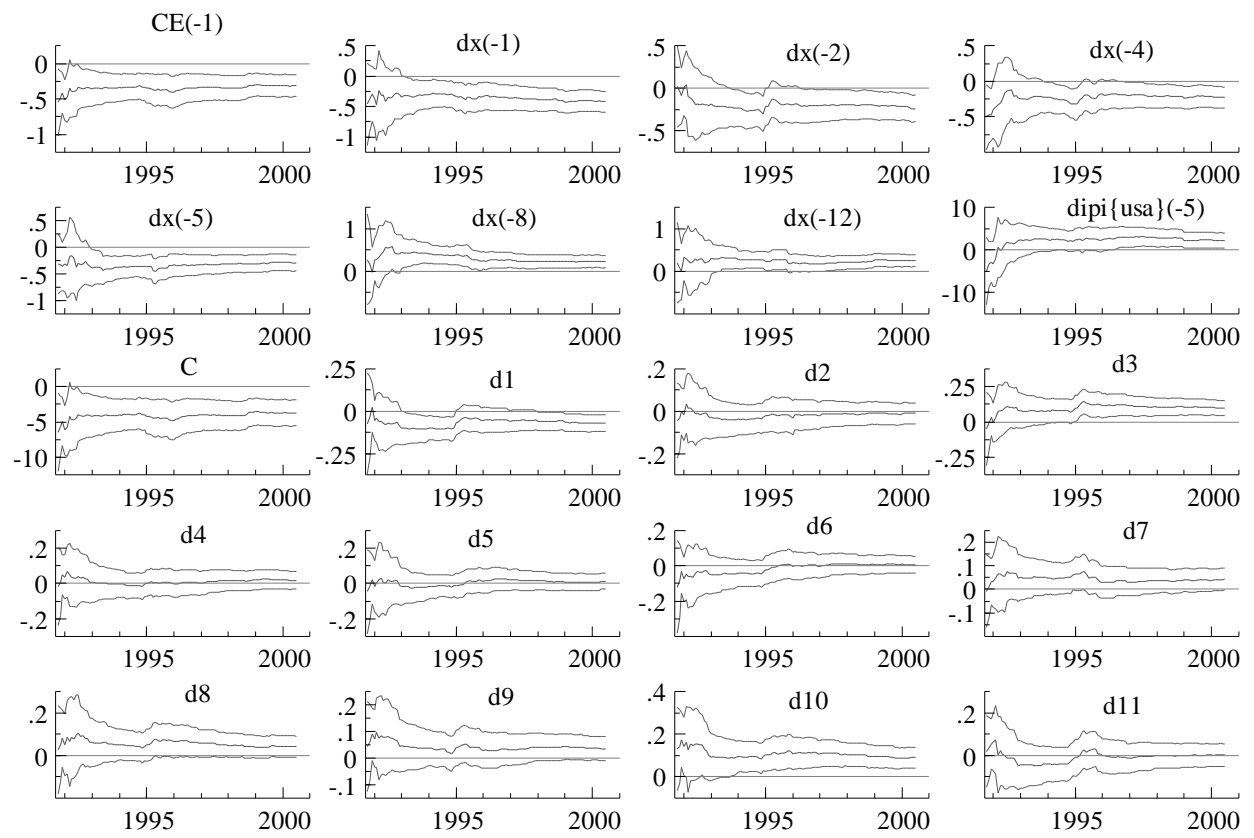

Se puede ver que los coeficientes de corto plazo también son relativamente constantes.

En el Cuadro 9 se presentan los resultados para las importaciones y contiene el mismo conjunto de estadísticos que el Cuadro 8. 
Cuadro 9

Modelos de Corrección de Error para las Importaciones

\begin{tabular}{|c|c|c|c|c|c|c|}
\hline \multirow[t]{2}{*}{ Variable } & \multicolumn{6}{|c|}{ Modelos } \\
\hline & $\Delta \mathrm{m}$ & $\Delta \mathrm{m}^{\{\text {cap }\}}$ & $\Delta \mathrm{m}^{\{\text {cons }\}}$ & $\Delta \mathrm{m}^{\{\text {int }\}}$ & $\Delta \mathrm{m}^{\{\text {int } \mathrm{Q}\}}$ & $\Delta \mathrm{m}^{\{\text {int-Q }\}}$ \\
\hline $\operatorname{Cem}_{\mathrm{t}-1}{ }^{\{\mathrm{i}\}}$ & $\begin{array}{c}-0.246 \\
(0.057) \\
\end{array}$ & $\begin{array}{c}-0.343 \\
(0.076) \\
\end{array}$ & $\begin{array}{l}-0.323 \\
(0.063) \\
\end{array}$ & $\begin{array}{l}-0.313 \\
(0.069) \\
\end{array}$ & $\begin{array}{c}-0.277 \\
(0.080)\end{array}$ & $\begin{array}{c}-0.331 \\
(0.064) \\
\end{array}$ \\
\hline$\Delta \mathrm{m}^{\{\mathrm{i}\}}{ }_{\mathrm{t}-1}$ & & $\begin{array}{c}-0.214 \\
(0.067)\end{array}$ & & & $\begin{array}{l}-0.440 \\
(0.082)\end{array}$ & \\
\hline$\Delta \mathrm{m}^{\{\mathrm{i}\}}{ }_{\mathrm{t}-2}$ & & & & & $\begin{array}{c}-0.447 \\
(0.089)\end{array}$ & \\
\hline$\Delta \mathrm{m}^{\{\mathrm{i}\}}{ }_{\mathrm{t}-3}$ & & & & & $\begin{array}{c}-0.374 \\
(0.093)\end{array}$ & \\
\hline$\Delta \mathrm{m}^{\{\mathrm{i}\}}{ }_{\mathrm{t}-4}$ & & & & & $\begin{array}{c}-0.263^{\circ o} \\
(0.078)\end{array}$ & \\
\hline$\Delta \mathrm{m}^{\{\mathrm{i}\}}{ }_{\mathrm{t}-5}$ & & & & & $\begin{array}{c}-0.195 \\
(0.064)\end{array}$ & \\
\hline$\Delta \mathrm{m}^{\{\mathrm{i}\}}{ }_{\mathrm{t}-7}$ & $\begin{array}{c}0.101 \\
(0.035) \\
\end{array}$ & & & & & \\
\hline$\Delta \mathrm{m}^{\{\mathrm{i}\}}{ }_{\mathrm{t}-10}$ & $\begin{array}{l}-0.139 \\
(0.037)\end{array}$ & & $\begin{array}{c}-1.888 \\
(0.060)\end{array}$ & $\begin{array}{l}-0.130 \\
(0.044)\end{array}$ & & \\
\hline$\Delta \mathrm{x}$ & $\begin{array}{l}0.783^{\circ o} \\
(0.043)\end{array}$ & & & $\begin{array}{c}0.914 \\
(0.048)\end{array}$ & $\begin{array}{l}1.006^{\mathrm{oo}} \\
(0.088)\end{array}$ & $\begin{array}{c}0.510 \\
(0.054)\end{array}$ \\
\hline$\Delta$ ivpi & $\begin{array}{c}0.670 \\
(0.091) \\
\end{array}$ & $\begin{array}{c}1.605 \\
(0.201) \\
\end{array}$ & $\begin{array}{c}1.772 \\
(0.205) \\
\end{array}$ & & & $\begin{array}{c}1.221 \\
(0.122) \\
\end{array}$ \\
\hline$\Delta \mathrm{tcr}_{\mathrm{t}}$ & & & $\begin{array}{c}-0.487 \\
(0.156)\end{array}$ & & & \\
\hline$\Delta \mathrm{tcr}_{\mathrm{t}-1}$ & $\begin{array}{l}-0.199 \\
(0.057)\end{array}$ & $\begin{array}{c}-0.412 \\
(0.161)\end{array}$ & & $\begin{array}{c}-0.218 \\
(0.076)\end{array}$ & & $\begin{array}{c}-0.370 \\
(0.077)\end{array}$ \\
\hline$\Delta \mathrm{tcr}_{\mathrm{t}-2}$ & & & & & & $\begin{array}{c}0.175 \\
(0.072)\end{array}$ \\
\hline$\Delta \mathrm{tcr}_{\mathrm{t}-3}$ & & & & & $\begin{array}{c}0.342 \\
(0.101)\end{array}$ & \\
\hline Est. MCE & -5.460 & -6.850 & -5.200 & -6.370 & -4.810 & -6.820 \\
\hline $\mathrm{R}^{2}$ & 0.942 & 0.740 & 0.813 & 0.903 & 0.901 & 0.892 \\
\hline $\mathrm{R}^{2}$ Ajust. & 0.932 & 0.705 & 0.787 & 0.888 & 0.881 & 0.875 \\
\hline E.E. & 0.023 & 0.067 & 0.069 & 0.029 & 0.040 & 0.031 \\
\hline Coef. Asimetría & -0.095 & 0.056 & 0.239 & -0.335 & -0.103 & -0.427 \\
\hline Curtosis & 3.617 & 2.890 & 3.484 & 3.514 & 2.440 & 3.144 \\
\hline Est. Jarque-Bera & 1.959 & 0.130 & 2.430 & 3.390 & 1.691 & 3.537 \\
\hline $\mathrm{LM}_{12} \mathrm{AR}$ & 1.555 & 1.533 & 0.839 & 0.695 & 1.042 & 1.106 \\
\hline $\mathrm{LM}_{12} \mathrm{ARCH}$ & 0.701 & 0.779 & 0.888 & 1.096 & 0.919 & 0.540 \\
\hline Prueba de White & 1.065 & 0.956 & 1.459 & 1.169 & $1.570 *$ & 0.72 \\
\hline RESET & 0.074 & 2.453 & 0.033 & 0.089 & 2.456 & 2.452 \\
\hline Chow B. (94:01) & $2.113 * *$ & 1.183 & 1.201 & $2.209 * *$ & $1.913 * *$ & $1.850 * *$ \\
\hline Chow Pred.( 94:01) & $1.782 *$ & 0.533 & 0.625 & 0.938 & 1.271 & 0.999 \\
\hline
\end{tabular}

$\mathrm{Cem}_{\mathrm{t}-1}=\mathrm{m}_{\mathrm{t}-1}-1.124 \mathrm{ivpi}_{\mathrm{t}-1}+0.283$ tcr $_{\mathrm{t}-1}-0.461 \mathrm{x}_{\mathrm{t}-1}$

$\mathrm{Cem}_{\mathrm{t}-1}{ }^{\text {'cap\} }}=\mathrm{m}_{\mathrm{t}-1}{ }^{\text {\{cap\} }}-1.966 \mathrm{ivpi}_{\mathrm{t}-1}+0.734 \mathrm{tcr}_{\mathrm{t}-1}$

$\mathrm{Cem}_{\mathrm{t}-1}^{\text {\{intt }}=\mathrm{m}_{\mathrm{t}-1}^{\text {|intt }}+0.476 \mathrm{tcr}_{\mathrm{t}-1}-0.908 \mathrm{x}_{\mathrm{t}-1}$

$\mathrm{Cem}_{\mathrm{t}-1}{ }^{\text {\{cons\} }}=\mathrm{m}_{\mathrm{t}-1}{ }^{\text {\{cons }\}}-1.753$ ivpi $_{\mathrm{t}-1}+1.160$ tcr $_{\mathrm{t}-1}$

$\mathrm{Cem}_{\mathrm{t}-1}{ }^{\{\mathrm{int}-\mathrm{Q}\}}=\mathrm{m}_{\mathrm{t}-1}{ }^{\{\mathrm{int}-\mathrm{Q}\}}+0.206 \mathrm{tcr}_{\mathrm{t}-1}-1.224 \mathrm{x}_{\mathrm{t}-\mathrm{t}}$

$\mathrm{Cem}_{\mathrm{t}-1}{ }_{\text {[int_QP) }}=\mathrm{m}_{\mathrm{t}-1}{ }^{\text {(int_QP) }}-1.200 \mathrm{ivpi}+0.146 \mathrm{tcr}_{\mathrm{t}-1}-0.323 \mathrm{x}_{\mathrm{t}-1}$ 
Los coeficientes de velocidad de ajuste para las importaciones también implican convergencia rápida. La presencia de términos de corto plazo refuerza esta propiedad. Los estadísticos ECM son todos significativos al 1\%. Las medidas de bondad de ajuste son similares a las encontradas para las exportaciones. No existen problemas con los residuales. Las pruebas RESET son fácilmente superadas en todos los casos. Sin embargo, solamente las ecuaciones para bienes de consumo y bienes de capital pasan las pruebas de Chow. El rechazo de la estabilidad con ambas pruebas ocurre solamente en cuanto a las importaciones totales.

En la Gráfica 11 se completa el análisis del modelo de corrección de error para las importaciones totales. En los paneles se presentan los estadísticos recursivos.

\section{Gráfica 11}

\section{Pruebas Recursivas de Estabilidad para el Modelo de las Importaciones Totales}
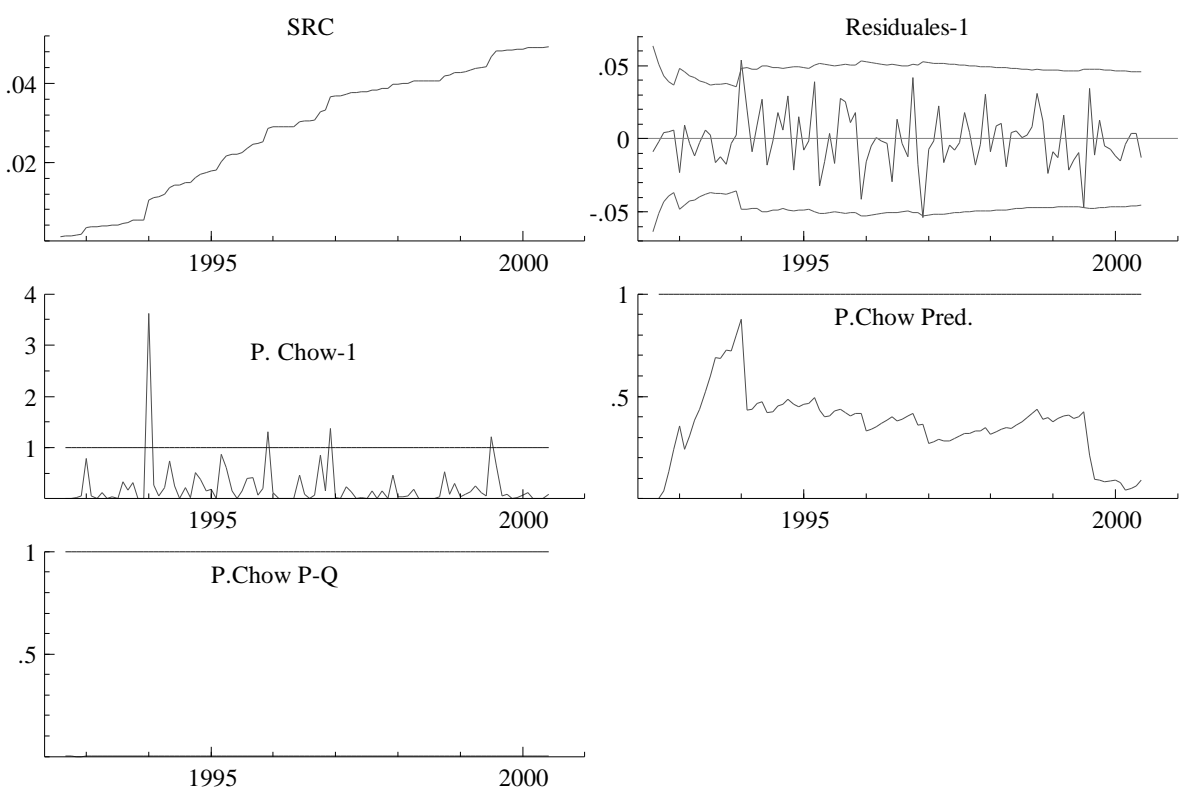

La suma de residuales se aproxima a una línea recta. Los residuales de un paso permanecen dentro de las bandas excepto en un mes. La secuencia de estadísticos de Chow de un paso muestra más observaciones extremas que en el caso de las exportaciones. Sin embargo, los estadísticos de Chow de n-pasos no señalan problema alguno. Para completar el análisis, en la Gráfica 12 se muestran las secuencias de estadísticos recursivos para el mismo modelo, los cuales parecen ser constantes a través de todo el período de muestra. 


\section{Gráfica 12}

Coeficientes Recursivos para el Modelo de las Importaciones Totales
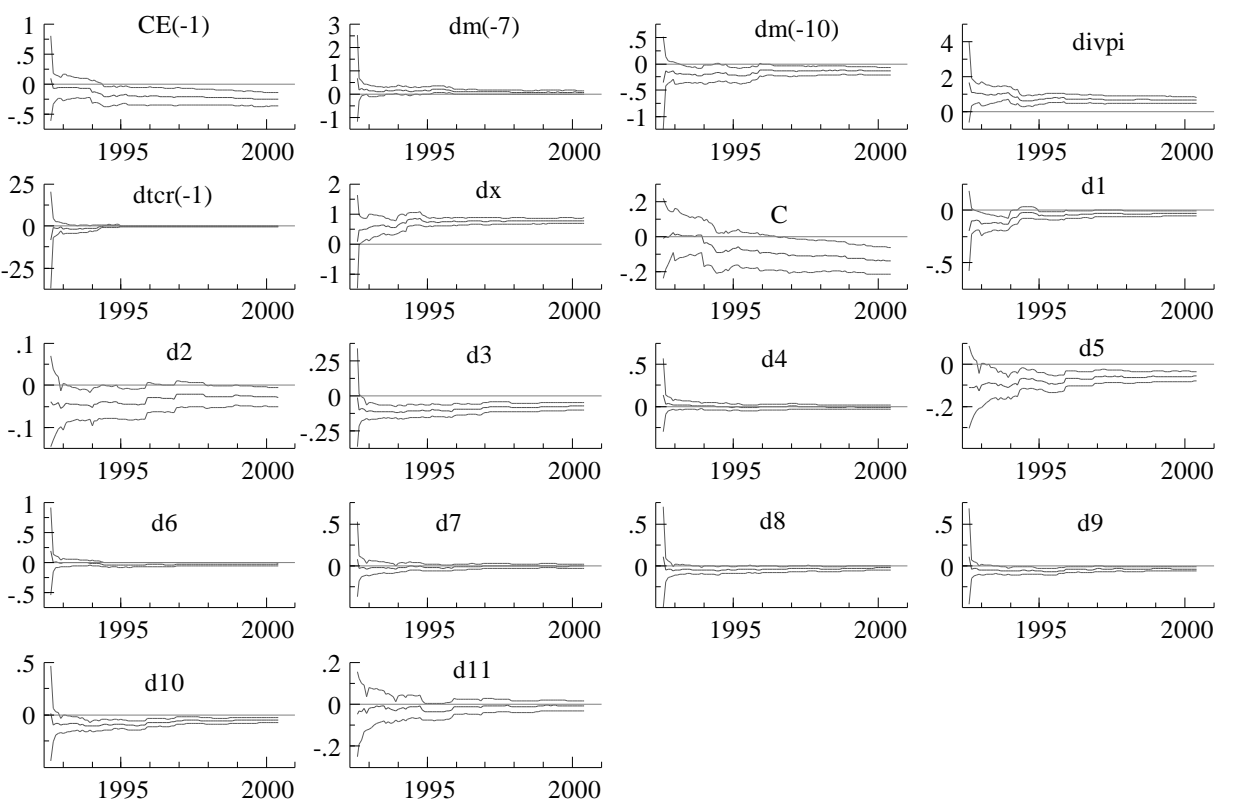

\section{Conclusiones}

El comercio exterior mexicano ha tenido un notable desempeño desde la segunda mitad de los noventa respecto, a las exportaciones han triplicado su tasa de crecimiento y fueron las más vigorosas en todo el mundo durante el período examinado. Se estimaron funciones de importaciones y exportaciones de largo plazo para el período 1990-2000 y se obtuvieron coeficientes que tienen los signos y magnitudes apropiadas. Se encontró que la ecuación de exportaciones, como una función del índice de la producción industrial de los Estados Unidos y el tipo de cambio real, es sumamente estable para dicho período. La demanda de importaciones totales, como función del índice de la producción industrial de México, del tipo de cambio real y de las exportaciones, muestra inestabilidad para el mismo período. No obstante, la ecuación de largo plazo ajusta los datos bastante bien aun sin considerar la estacionalidad ni la dinámica de corto plazo.

El análisis se extendió a los datos desagregados y para el período 1980-2000. Se demostró que solamente en unos pocos casos se puede encontrar un vector de cointegración para toda la muestra aunque, de acuerdo a las pruebas de Hansen (1992) para regresiones con variables I(1), ninguno de ellos fue estable. Sin embargo, en casi todos los casos, excepto uno, se encontró un vector de cointegración estable para el subperíodo 1990-2000. 
Para la mayoría de los casos, un vector de cointegración también fue encontrado para la submuestra 1980-1989 aunque ese vector no muestra la estabilidad característica del segundo segmento de la muestra. También se obtuvieron modelos de corrección de error para el período 1990-2000 y se encontró que éstos eran muy estables.

Sin la apertura comercial de los ochenta no habría ocurrido un cambio estructural tan importante y el éxito del comercio exterior mexicano no habría sido posible. En este sentido, el TLCAN puede ser interpretado como el evento que consolidó un proceso que venía desde la década previa con la entrada de México al GATT y que permitió la continuación de los beneficios de la apertura comercial. 


\section{Bibliografía}

Campos, J. and N. R. Ericsson, (2000) "Constructive data mining: Modelling consumer's expenditure in Venezuela," Board of Governors of the Federal Reserve System, International Finance Discussion Papers, April.

De Brouwer, Gordon y Neil R. Ericsson (1998) "Modelling Inflation in Australia," Journal of Business \& Economic Statistics, Octubre 1998, Vol. 16, No. 4.

Ericsson, Neil R. and James G. MacKinnon, "Distributions of Error Correction Tests for Cointegration," International Finance Discussion Paper No. 655, Board of Governors of the Federal Reserve System, 1999.

Gregory, A.W. y Hansen, B.E. (1996). "Residual-based Tests for Cointegration in Models with Regime Shifts," \{\}

Gould, David M., "Has NAFTA Changed North America Free Trade," Economic Review, Federal Reserve Bank of Dallas, First Quarter 1998.

Hansen, B.E. (1992) “Tests for Parameter Instability in Regressions with I(1) Processes,” Journal of Business and Economic Statistics, 10, 321-335.

Hao, K. (1996) "Testing for Structural Change in Cointegrated Regression Models: Some Comparisons and Generalizations," Econometric Reviews, 15, 401-429.

Hatanaka,M. (1996) “Time-Series-Based Econometrics,” Oxford University Press.

Johansen, S. y K. Juselius (1992) "Testing Structural Hypothesis in a Multivariate Cointegration Analysis of the PPP and UIP for the UK," Journal of Econometrics, 53, 211-244.

Krueger, A. O., (1999) "Trade Creation and Trade Diversion Under NAFTA," NBER Working Paper No. 7429, December 1999.

Kwiatkowski, D., P. Phillips, P. Schmidt and Y. Shin (1992): Testing the Null Hypothesis of Stationarity Against the Alternative of a Unit Root, Journal of Econometrics 54, 159-178.

Phillips, P.C.B. y Bruce E. Hansen (1990), "Statistical Inference in Instrumental Variables Regression With I(1) Processes," Review of Economic Studies, 57, 99-125. 
Phillips P.C.B. y C.E. Quintos (1993), "Parameter Constancy in Cointegrating Regressions," Empirical Economics, 18, 675-706.

Rao, B.B. (1994) “Cointegration”, St. Martin's Press.

Reinhart, C., 1995, "Devaluation, Relative Prices, and International Trade", IMF Staff Papers, vol.42, No 2 .

Rose, A., 1991, "The Role of Exchange Rates in Popular Models of International Trade: Does the Marshall-Lerner Condition Hold?” Journal of International Economics, vol. 30, 301-16.

Senhadji, A. (1998), "Time Series Estimation of Structural Import Demand Equations: A CrossCountry Analysis", IMF Staff Papers, vol. 45, June.

Senhadji, A. and C. Montenegro (1998), "Time Series Analysis of Export Demand Equations: A Cross-Country Analysis", IMF Working Paper, 98/149, October. 